TRANSACTIONS OF THE

AMERICAN MATHEMATICAL SOCIETY

Volume 351, Number 10, Pages 3989-4026

S 0002-9947(99)02256-4

Article electronically published on February 8, 1999

\title{
CHERN CLASSES FOR SINGULAR HYPERSURFACES
}

\author{
PAOLO ALUFFI
}

\begin{abstract}
We prove a formula expressing the Chern-Schwartz-MacPherson class of a hypersurface in a nonsingular variety as a variation on another definition of the homology Chern class of singular varieties, introduced by W. Fulton; and we discuss the relation between these classes and others, such as Mather's Chern class and the $\mu$-class we introduced in previous work.
\end{abstract}

\section{INTRODUCTION}

There are several candidates for a notion of homology 'Chern class' of a (possibly singular) algebraic variety $X$, agreeing with the class obtained as dual of the total Chern class of the tangent bundle of $X$ if $X$ is nonsingular. One such notion was introduced by R. MacPherson [MacPherson], agreeing (as it was later understood) with a class introduced earlier by M.-H. Schwartz, and enjoying good functorial properties. A different class was defined by W. Fulton ([Fulton], 4.2.6), in a different and somewhat more general setting. The main purpose of this paper is to prove a precise relation between the Schwartz-MacPherson and the Fulton class of a hypersurface $X$ of a nonsingular variety. We denote these two classes by $c_{\mathrm{SM}}(X)$, $c_{F}(X)$ respectively.

A summary of the context and essential definitions is given in $\S 1$, where we present several different statements of the result. The version which expresses most directly the link between the two classes mentioned above goes as follows. Fulton's class for a subscheme $X$ of a nonsingular variety $M$ is defined by

$$
c_{F}(X):=c\left(\left.T M\right|_{X}\right) \cap s(X, M),
$$

that is, by capping the total Chern class of the ambient space against the Segre class of the subscheme (it is proved in [Fulton], 4.2.6 that this definition does not depend on the choice of the ambient variety $M$ ). For a hypersurface $X$ of a nonsingular variety $M$, let $Y$ be the 'singular subscheme' of $X$ (locally defined by the partial derivatives of an equation of $X)$. For an integer $k \geq 0$ we can consider the $k$-th thickening $X^{(k)}$ of $X$ along $Y$, and then consider its Fulton class $c_{F}\left(X^{(k)}\right)$. It is easily seen that $c_{F}\left(X^{(k)}\right)$ is a polynomial in $k$ (with coefficients in the Chow group $\left.A_{*}(X)\right)$, so it can be formally evaluated at negative $k$ 's. We can then define a class

$$
c_{*}(X):=c_{F}\left(X^{(-1)}\right) .
$$

The main result of this paper is

Theorem. $c_{*}(X)$ is equal to the Chern-Schwartz-MacPherson class of $X$.

Received by the editors June 3, 1997.

1991 Mathematics Subject Classification. Primary 14C17, 32S60.

Supported in part by NSF grant DMS-9500843.

(C)1999 American Mathematical Society 
This result is quite distant in spirit from both Schwartz's original definition of $c_{\mathrm{SM}}(X)$, which depends on a suitable stratification of $X$, and MacPherson's definition, which involves adding Chern-Mather classes of subvarieties of $X$ weighted according to their own singularity, as measured by 'local Euler obstructions'. The formula seems at first equally distant from the good functoriality properties which make $c_{\mathrm{SM}}$ effectively computable: as pointed out in [Fulton], p. 377, functoriality properties of $c_{F}$ are somewhat elusive. However, our proof will precisely consist of showing that the 'twisted' $c_{F}$ does enjoy enough of these functoriality properties to force it to equal $c_{\mathrm{SM}}$.

We do not know whether our result is an essential feature of hypersurfaces, or whether a formula similar to $\left(^{*}\right)$ may compute the Schwartz-MacPherson class of arbitrary varieties. While this is a natural question, the approach of this paper does not seem well suited to address it. One reason for presenting several different formulations of the result is indeed that we do not see at the moment which formulation is more suited to be generalized to arbitrary varieties.

These statements are given in $\S 1$, Theorem I.1-5, where we offer several alternative formulations for the class $c_{*}(X)$. More specifically:

- An explicit form in terms of Segre classes is given in $\$ 1.1$.

- In $\S 1.2$ we give the form presented above. This was conjectured in [Aluffi2], where we put restrictions on the line bundle $\mathcal{L}=\mathcal{O}(X)$ of $X$ and we could only prove the conjectured equality 'numerically', that is, after taking degrees with respect to $\mathcal{L}$. The result proved in this note holds in the Chow group of $X$, and without hypotheses on $\mathcal{O}(X)$.

- In $\S 1.3$ we discuss a form which attempts to put under the same umbrella the Schwartz-MacPherson class together with the Fulton and Chern-Mather classes, by expressing all in terms of natural classes on the blow-up of the ambient variety along the singular subscheme of $X$.

- In $\S 1.4$ we introduce notations which we use elsewhere and in the proof of the main theorem, and give a formulation of the statement in term of these notations;

- Finally, in $\S 1.5$ we express $c_{*}(X)$ as an explicit formula in terms of $\mu$-class we introduced in [Aluffi1]. In this form, and using the notations introduced in $\S 1.4$, the result is

$$
c_{\mathrm{SM}}(X)=c_{F}(X)+c(\mathcal{L})^{\operatorname{dim} X} \cap\left(\mu_{\mathcal{L}}(Y)^{\vee} \otimes_{M} \mathcal{L}\right),
$$

and therefore it interprets the $\mu$-class essentially as the difference between $c_{F}(X)$ and $c_{\mathrm{SM}}(X)$. In fact this is the form in which we first produced the result; the key technical step in proving the main result of this paper can be written as a formula describing the behavior of the $\mu$-class under blow-ups (see $\S 4.2$ ).

The equivalence of the different formulations is straightforward and is proved along the way in $\S 1$. The proof of the main result is in $\S \S 2$ and 3. We show that a class satisfying the functorial properties prescribed by Grothendieck and Deligne (which motivated MacPherson's work) must necessarily agree with $c_{*}(X)$; the Schwartz-MacPherson class $c_{\mathrm{SM}}(X)$ is such a class. Our proof uses resolution of singularities, reducing the statement to a computation for a divisor with normal crossing ( $(2)$, and to studying the behavior of the class under blow-ups along nonsingular centers ( $\S 3)$. This ultimately involves a rather detailed analysis of an adaptation of MacPherson's graph construction applied to our situation: the required equality is expressed in terms of the vanishing of the contribution of a 'cycle 
at infinity' obtained in the construction, and this contribution can be evaluated explicitly.

While this proof is elementary, and tailored for our set-up (Chow groups of algebraic varieties over a field of characteristic 0 ), it is admittedly rather long and involved for what it aims to show. We sketch a much shorter argument in $\S 1.6$, which hinges on the index formula of [BDK]. This reduces the proof to the computation of a characteristic cycle (see the Fact in $\S 1.6$ ), which we leave to the expert reader. This alternative argument relies on a more powerful framework than the longer proof given in $\S \S 2$ and 3, and in a sense clarifies 'why' the main theorem is true. We suspect that a suitable generalization of the result to arbitrary singular varieties will necessarily have to employ this more powerful technology.

I am very grateful to the referee of a previous version of this work for detailed suggestions leading to the present $§ 1.6$.

$\S 4$ collects a few consequences of the main theorem. For example, in its raw form (presented in $\S 1.1$ ), the result writes $c_{\mathrm{SM}}(X)$ as a certain combination of Segre classes of the singular subscheme of $X$ in the ambient variety $M$. The good functoriality properties of $c_{\mathrm{SM}}(X)$ then translate into properties of these Segre classes, which often we are not able to prove more directly. We give one (rather technical) example of these phenomena in $\S 4.3$.

Properties of Segre classes frequently have concrete geometric applications. Here is a simple example of such an application, which can be proved easily as a consequence of the main theorem (see $\S 4.4$ for details):

Proposition. Let $M_{1}, M_{2}$ be two hypersurfaces in $\mathbb{P}^{N}$, and assume the contact scheme of $M_{1}$ and $M_{2}$ is nonsingular (as a scheme) and positive-dimensional. Then $\operatorname{deg} M_{1}=\operatorname{deg} M_{2}$.

Here the 'contact scheme' is the singularity subscheme of $M_{1} \cap M_{2}$ (in the above sense), a scheme supported on the locus where $M_{1}$ and $M_{2}$ are tangent. This corollary thus states that if two hypersurfaces of projective space are tangent - in the strong 'schemey' sense specified above - along a nonsingular positive-dimensional locus, then their degree must be equal: to our knowledge, this observation is new (although not difficult to prove otherwise: Robert Varley kindly showed us an alternative argument).

The paper is organized so that a hasty reader who is willing to trust us on the main technical step of the elementary proof can skip $\S 3$ at first: the notations introduced in $\S 3$ are not used elsewhere. In fact, an even more trusting reader could skip all but the beginning of $\S 2$. The experts will probably be content with the sketch of the alternative proof given in $\S 1.6$, and will want to stop there.

Notations are gravely abused in this paper (pull-backs are usually omitted, etc.). The worst abuse occurs when we ask the reader to interpret a class defined a priori on an ambient variety $M$, but supported on a subvariety $X$, as a class defined on $X$ : this will mean that there is only one reasonable way to interpret the class as a push-forward of a class from $A_{*} X$, and that the given formula is a short-hand for the latter class. This saves us a great amount of notational grief, especially in $\S 3$; the reader who feels uncomfortable about this choice will only believe our result after push-forward to the ambient variety $M$.

I thank Barbara Fantechi, William Fulton, Roberto Silvotti, and Tatsuo Suwa for useful comments. 


\section{Statements of the RESUlt}

1.1. Chern-Schwartz-MacPherson class and Segre classes. A constructible function on an algebraic variety $X$ is an integral linear combination of characteristic functions of closed subvarieties. Over the complex numbers one can define a functor assigning to $X$ the group of constructible functions on $X$; for any proper morphism $f: X \rightarrow Y$, the push-forward $f_{*}$ is defined by setting

$$
f_{*}\left(1_{V}\right)(p)=\chi\left(f^{-1}(p) \cap V\right)
$$

for $V$ a subvariety of $X$ and $p \in Y$, and extending by linearity; here $\chi$ denotes topological Euler characteristic. Grothendieck and Deligne conjectured, and MacPherson proved ([MacPherson]), that there exists a natural transformation from this functor to homology, which, for $X$ nonsingular, sends the constant $1_{X}$ to the total homology Chern class of $X$

$$
c(T X) \cap[X] .
$$

It is then natural to consider the image of $1_{X}$ for arbitrary $X$; we call this class the (Chern-)Schwartz-MacPherson class of $X$, denoted $c_{\mathrm{SM}}(X)$, as the class was later shown to agree with a class previously defined by M.-H. Schwartz ([Schwartz, B-S]). After work of C. Sabbah and G. Kennedy ([Kennedy]), this definition can be extended to varieties over arbitrary fields of characteristic 0 ; and $c_{\mathrm{SM}}(X)$ lives in the Chow group $A_{*} X$ of cycles modulo rational equivalence. Also, for possibly nonreduced $X$, we define $c_{\mathrm{SM}}(X)=c_{\mathrm{SM}}\left(X_{\text {red }}\right)$.

The main theorem in this paper will give explicit formulas for $c_{\mathrm{SM}}(X)$, in the case where $X$ is a hypersurface of an $n$-dimensional nonsingular variety $M$, that is, the zero-scheme of a nonzero section $F$ of a line bundle $\mathcal{L}$ on $M$. We give the result in its raw form in terms of Segre classes here in $\S 1.1$; however, the result is more significant if the formula is rewritten to emphasize its relation with other definitions of characteristic classes for singular hypersurfaces. This is done in the rest of $\S 1$. In $\S 1.4$ we will also introduce a notation which makes some of the formulas easier to handle. A quick sketch of an argument proving the main theorem is presented in $\S 1.6$; a complete and elementary (but longer) proof is given in $\S \S 2$ and 3.

Assume $c_{\mathrm{SM}}$ satisfies the above functoriality condition, and assume resolution of singularities à la Hironaka holds (for example, $c_{\mathrm{SM}}$ can be the Schwartz-MacPherson class, in characteristic zero). By the singularity (sub)scheme of a hypersurface $X \subset M$ as above we mean the subscheme of $M$ defined locally by $F$ and its first partial derivatives - that is, by the jacobian ideal of $X$. (Note: the definition of singularity scheme of $X$ given in [Aluffi1], §1.1, fails to include $F$ among the local generators of its ideal. The definition given here is the 'correct' one; all the results in [Aluffi1] hold for this notion.) The singular scheme of $X$ depends on $X$ only, and not on a specific realization of $X$ as a hypersurface of a nonsingular variety.

For $Y \subset X$, we define a class $s(X \backslash Y, M) \in A_{*} X$ by setting its dimension- $m$ component to be

$$
s(X \backslash Y, M)_{m}=s(X, M)_{m}+(-1)^{n-m} \sum_{j=0}^{n-m}\left(\begin{array}{c}
n-m \\
j
\end{array}\right) X^{j} \cdot s(Y, M)_{m+j}
$$

(here and in the following, $s(Y, M)$ denotes the Segre class of $Y$ in $M$ in the sense of [Fulton], Chapter 4). 
Theorem I.1. Let $X$ be a hypersurface in a nonsingular variety $M$, and let $Y$ be its singular scheme. Then $c_{S M}(X)=c_{*}(X)$, where

$$
c_{*}(X)=c(T M) \cap s(X \backslash Y, M) .
$$

The reader will notice a similarity among the class $s(X \backslash Y, M)$ defined above and formulas for residual intersections (cf. [Fulton], §9.2). Of course this is not accidental. The connection will be clarified in the next subsection.

1.2. Chern-Schwartz-MacPherson class and Fulton's Chern class. Let $X$ be a scheme embeddable in a nonsingular variety $M$. Fulton shows ([Fulton], 4.2.6) that the class

$$
c(T M) \cap s(X, M)
$$

only depends on $X$ (and not on the choice of the ambient variety $M$ ). We will call this class Fulton's Chern class of $X$, denoted $c_{F}(X)$.

Now let $X$ again denote a divisor in a nonsingular variety $M$ of dimension $n, \mathcal{L}$ its line bundle, and $Y$ its singular subscheme. Fix an integer $k \geq 0$ and consider the scheme $X^{(k)}$ obtained by 'thickening $X k$ times along $Y$ ': more precisely, if $\mathcal{I}_{Y}$ denotes the ideal of $Y$ and $\mathcal{J}$ is the locally principal ideal of $X$, then $X^{(k)}$ is the subscheme of $M$ defined by the ideal $\mathcal{J} \cdot \mathcal{I}_{Y}^{k}$. We may then consider the class

$$
c_{F}\left(X^{(k)}\right)
$$

in $A_{*} X$. We observed in [Aluffi2] that this class is a polynomial in $k$ with coefficients in $A_{*} X$, so it can be formally evaluated for arbitrary $k$. It is also clear from the definition that

$$
c_{F}(X)=c_{F}\left(X^{(0)}\right)
$$

The main theorem of this note can then be stated as:

Theorem I.2. Let $X$ be a hypersurface in a nonsingular variety $M$, and let $c_{F}\left(X^{(k)}\right)$ be defined as above. Then $c_{S M}(X)=c_{*}(X)$, where

$$
c_{*}(X)=c_{F}\left(X^{(-1)}\right) .
$$

To see that this statement is equivalent to the one given in $\S 1.1$ amounts to applying standard residual intersection formulas to compute $s\left(X^{(k)}, M\right)$ : by Proposition 9.2 in [Fulton], the $m$-dimensional component of this class is

$$
s\left(X^{(k)}, M\right)_{m}=s(X, M)_{m}+\sum_{j=0}^{n-m}\left(\begin{array}{c}
n-m \\
j
\end{array}\right)(-X)^{j} \cdot k^{n-m-j} s(Y, M)_{m+j} .
$$

That is, $s\left(X^{(-1)}, M\right)$ is the class $s(X \backslash Y, M)$ introduced above, and it follows that the two expressions for $c_{*}(X)$ in the two statements of the theorem agree.

1.3. Chern-Schwartz-MacPherson class and Mather's Chern class. Another notion of Chern classes for possibly singular varieties can be defined as follows. For a reduced pure-dimensional $X$ embedded in a nonsingular $M$, let $X^{\circ}$ denote the nonsingular part of $X$. The Nash blow-up NB of $X$ is the closure in $\operatorname{Grass}_{\operatorname{dim} X}\left(\left.T M\right|_{X}\right)$ of the image of the map associating with every $p \in X^{\circ}$ the tangent space to $X^{\circ}$ at $p$; it comes equipped with a natural map $\pi$ to $X$. The 
universal subbundle $\mathcal{T}$ of $\operatorname{Grass}_{\operatorname{dim} X}\left(\left.T M\right|_{X}\right)$ restricts to $T X^{\circ}$ over $X^{\circ} \subset N B(X)$, so it is rather natural to consider the class

$$
\pi_{*}(c(\mathcal{T}) \cap[X]) .
$$

Again, one proves this is independent of the choice of $M$; this class is called Mather's Chern class of $X$, and we will denote it $c_{M}(X)$. MacPherson computes $c_{\mathrm{SM}}(X)$ as a suitable combination of Mather's Chern classes of subvarieties of $X$.

The definition of $c_{M}(X)$ can be rewritten in a slightly different way. If $X$ is a reduced hypersurface in $M, x_{1}, \ldots, x_{n}$ are local parameters for $M$, and $F=0$ is a local equation for $X$, then $\operatorname{Grass}_{\operatorname{dim} X}(T M)=\mathbb{P} T^{*} M$, the projectivized cotangent bundle to $M$, and the map from $X^{\circ}$ is written in natural coordinates

$$
p \mapsto\left(p ;\left.\frac{\partial F}{\partial x_{1}}\right|_{p}: \cdots:\left.\frac{\partial F}{\partial x_{n}}\right|_{p}\right) .
$$

It follows that locally $N B$ can be recovered as the blow-up of $X$ along the ideal of partials of the section of $\mathcal{L}=\mathcal{O}(X)$ defining $X$ in $M$ (cf. also [Nobile], where a corresponding observation is worked out for more general $X)$. We need a global version of this observation.

Lemma I.1. Let $X$ be a reduced hypersurface of a nonsingular variety $M$, and let $Y$ be the singular subscheme of $X$. Then NB is the proper transform of $X$ in the blow-up $B \ell_{Y} M \stackrel{\pi}{\longrightarrow} M$ of $M$ along $Y$. Further, there is a bundle $\mathcal{T}$ on $B \ell_{Y} M$ such that

$$
c_{M}(X)=\pi_{*}(c(\mathcal{T}) \cap[N B]) .
$$

Proof. Let $\mathcal{P}_{M}^{1} \mathcal{L}$ denote the bundle of principal parts of $\mathcal{L}$ over $M$, where $\mathcal{L}=\mathcal{O}(X)$ as above, and consider the section $M \rightarrow \mathcal{P}_{M}^{1} \mathcal{L}$ determined by $F \in H^{0}(M, \mathcal{L}) ;$ locally we can write this as

$$
p \mapsto\left(p ; F(p):\left.\frac{\partial F}{\partial x_{1}}\right|_{p}: \cdots:\left.\frac{\partial F}{\partial x_{n}}\right|_{p}\right)
$$

(recall that $\mathcal{P}_{M}^{1} \mathcal{L}$ fits into an exact sequence

$$
\left.0 \rightarrow T^{*} M \otimes \mathcal{L} \rightarrow \mathcal{P}_{M}^{1} \mathcal{L} \rightarrow \mathcal{L} \rightarrow 0\right) .
$$

It follows that the closure of the image of the corresponding rational map

$$
M \rightarrow \mathbb{P P}_{M}^{1} \mathcal{L}
$$

is the blow-up $B \ell_{Y} M \stackrel{\pi}{\longrightarrow} M$ along the singular scheme $Y$ of $X$ defined in $\S 1.1$. Over $X$ this reproduces (up to tensoring by $\mathcal{L}$ ) the map to $\mathbb{P} T^{*} M$ considered above, and the first part of the statement follows.

For the second part, let $\mathcal{Q}$ be the universal quotient bundle of $\mathbb{P P}_{M}^{1} \mathcal{L}$, and consider $\mathcal{T}=\mathcal{Q}^{\vee} \otimes \mathcal{L}$. Observe that the universal subbundle $\mathcal{O}(-1)$ of $\mathbb{P P}_{M}^{1} \mathcal{L}$ is $\subset T^{*} M \otimes \mathcal{L}$ over $N B$ (since this dominates $X$, and $F \equiv 0$ over $X$ ), so there is a sequence

$$
\left.\left.0 \rightarrow \mathcal{Q}^{\prime}\right|_{N B} \rightarrow \mathcal{Q}\right|_{N B} \rightarrow \mathcal{L} \rightarrow 0,
$$

where $\mathcal{Q}^{\prime}$ is the universal quotient bundle of $\mathbb{P}\left(\left.T^{*} M \otimes \mathcal{L}\right|_{X}\right)$. Chasing the identification

$$
\operatorname{Grass}_{n-1}\left(\left.T M\right|_{X}\right) \cong \mathbb{P}\left(\left.T^{*} M\right|_{X}\right) \cong \mathbb{P}\left(\left.T^{*} M \otimes \mathcal{L}\right|_{X}\right)
$$


shows that $\mathcal{Q}^{\prime \vee} \otimes \mathcal{L}$ is the bundle used above in the definition of $c_{M}$, and $\mathcal{T}=\mathcal{Q}^{\vee} \otimes \mathcal{L}$ differs from this by a trivial factor.

The next lemma gives the Mather class in an alternative form, which highlights a remarkable analogy with the Schwartz-MacPherson class. Still assuming $X$ reduced, in $B \ell_{Y} M$ we have the proper transform $N B$ of $X ; X$ itself pulls back to a Cartier divisor of $B \ell_{Y} M$, which we still denote $X$; also, we have the exceptional divisor $\mathcal{Y}$, and we note that $\mathcal{O}(\mathcal{Y})$ is the restriction of $\mathcal{O}(-1)$ from $\mathbb{P P}_{M}^{1} \mathcal{L}$.

Lemma I.2. With notations as above,

$$
c_{M}(X)=c(T M) \cap \pi_{*}\left(\frac{[N B]}{1+X-\mathcal{Y}}\right) .
$$

Proof. From the proof of Lemma I.1, we know that

$$
c_{M}(X)=\pi_{*}\left(c\left(\left(\frac{\mathcal{P}_{M}^{1} \mathcal{L}}{\mathcal{O}(-1)}\right)^{\vee} \otimes \mathcal{L}\right) \cap[N B]\right) .
$$

Because $c(\mathcal{L})=1+X, c(\mathcal{O}(1))=1-\mathcal{Y}$, and $c\left(\mathcal{P}_{M}^{1} \mathcal{L}\right)=c\left(T^{*} M \otimes \mathcal{L}\right) c(\mathcal{L})$, this gives

$$
\begin{aligned}
c_{M}(X) & =\pi_{*}\left(\frac{c\left(\left(\mathcal{P}_{M}^{1} \mathcal{L}\right)^{\vee} \otimes \mathcal{L}\right)}{1+X-\mathcal{Y}} \cap[N B]\right) \\
& =\pi_{*}\left(c\left(T M \otimes \mathcal{L}^{\vee} \otimes \mathcal{L}\right) c\left(\mathcal{L}^{\vee} \otimes \mathcal{L}\right) \cap \frac{[N B]}{1+X-\mathcal{Y}}\right) \\
& =c(T M) \cap \pi_{*}\left(\frac{[N B]}{1+X-\mathcal{Y}}\right) .
\end{aligned}
$$

With this understood, the third form of the result of this paper should appear more significant: it says that the Schwartz-MacPherson class can be obtained similarly to the Mather class, by replacing the class $[N B]$ of the proper transform of $X$ with the residual to the exceptional divisor in the whole inverse image of $X$. In other words, under the same hypotheses of the first and second statement of the main theorem:

Theorem I.3. Let $X$ be a hypersurface in a nonsingular variety $M$, and let $\pi, \mathcal{Y}$ be as defined above. Then $c_{S M}(X)=c_{*}(X)$, where

$$
c_{*}(X)=c(T M) \cap \pi_{*}\left(\frac{[X]-[\mathcal{Y}]}{1+X-\mathcal{Y}}\right) .
$$

Note: $X$ may be nonreduced here.

To see that Theorem I.3 is equivalent to the other statements, we have to show that

$$
\pi_{*}\left(\frac{[X]-[\mathcal{Y}]}{1+X-\mathcal{Y}}\right)=s(X \backslash Y, M) .
$$

One way to see this is to consider for all $k$ the class

$$
\pi_{*}\left(\frac{[X]+k[\mathcal{Y}]}{1+X+k \mathcal{Y}}\right):
$$


for $k \geq 0,[X]+k[\mathcal{Y}]$ is the cycle of the inverse image of the scheme $X^{(k)}$ considered in $\S 1.2$; hence, by the birational invariance of Segre classes,

$$
\pi_{*}\left(\frac{[X]+k[\mathcal{Y}]}{1+X+k \mathcal{Y}}\right)=s\left(X^{(k)}, M\right)
$$

for $k \geq 0$. Both sides are polynomials in $k$, so they must agree for $k=-1$, and this is what $(*)$ claims.

1.4. Notational device. The following notations will be helpful in writing the arguments needed in our proof of the theorem stated above. In fact they simplify considerably the first formulation we gave, by giving a summation-free alternative definition for the class $s(X \backslash Y, M)$.

Definition. If $A=\bigoplus_{i} a^{i}$ is a rational equivalence class on a scheme, indexed by codimension, we let

$$
A^{\vee}=\sum_{i \geq 0}(-1)^{i} a^{i}
$$

the dual of $A$; also, for a line bundle $\mathcal{L}$ we let

$$
A \otimes \mathcal{L}=\sum_{i \geq 0} \frac{a^{i}}{c(\mathcal{L})^{i}},
$$

the tensor of $A$ by $\mathcal{L}$. We put a subscript to $\otimes$ to denote the ambient in which the codimension is computed (which may be larger than the scheme on which the class lives), if this doesn't seem otherwise clear from the context.

These notations were introduced in [Aluffi2], where we also proved simple compatibilities with standard vector bundle operations (Prop. 1 and 2 in [Aluffi2], §2). We will freely use those properties in this note (especially in $\S 2$ ).

Lemma I.3. With notations as above,

$$
s(X \backslash Y, M)=s(X, M)+c(\mathcal{L})^{-1} \cap\left(s(Y, M)^{\vee} \otimes_{M} \mathcal{L}\right) .
$$

Proof. This is detailed in [Aluffi2] (section 2), so we will not reproduce it here. It is a good exercise for the reader interested in acquiring some familiarity with the notations introduced above: if $X \subset W \subset M$, with $X, M$ as above, and $R$ is the residual scheme to $X$ in $W$, show that

$$
s(W, M)=s(X, M)+c(\mathcal{L})^{-1} \cap\left(s(R, M) \otimes_{M} \mathcal{L}\right) .
$$

Applying to $X^{(k)}$ (as in $\S 1.2$ above) and setting $k=-1$ gives the statement. Details may be found in [Aluffi2].

By Lemma I.3, the main theorem can be formulated:

Theorem I.4. Let $X$ be a hypersurface in a nonsingular variety $M$, and let $Y$ be its singular scheme. Then $c_{S M}(X)=c_{*}(X)$, where

$$
c_{*}(X)=c(T M) \cap\left(s(X, M)+c(\mathcal{L})^{-1} \cap\left(s(Y, M)^{\vee} \otimes_{M} \mathcal{L}\right)\right) .
$$


1.5. Chern-Schwartz-MacPherson class and $\mu$-classes. Another formulation of the main result of this paper can be given in terms of the $\mu$-class introduced in [Aluffi1].

If $Y$ is a singular subscheme of a hypersurface $X$ in a nonsingular variety $M$, with $\mathcal{L}=\mathcal{O}(X)$, the $\mu$-class of $Y$ with respect to $\mathcal{L}$ is defined by

$$
\mu_{\mathcal{L}}(Y)=c\left(T^{*} M \otimes \mathcal{L}\right) \cap s(Y, M) .
$$

This class does not depend on the specific realization of $Y$ as a singular scheme of a hypersurface (Corollary 1.7 in [Aluffi1]). Note that if $Y \subset M$ is nonsingular, and with the notations introduced in $\S 1.4$,

$$
c(\mathcal{L})^{\operatorname{dim} M} \cap\left(\mu_{\mathcal{L}}(Y)^{\vee} \otimes_{M} \mathcal{L}\right)=(-1)^{\operatorname{codim}_{M} Y} c(T Y) \cap[Y] .
$$

(This isn't entirely obvious from the definition; it follows from Corollary 1.8 in [Aluffi1]. Of course this formula fails spectacularly unless one assumes $Y$ is realized as the singular scheme of a hypersurface in $M$.) That is, the $\mu$-class can be used to define yet another class extending the notion of Chern class to (certain) possibly singular varieties. It turns out that this class gives a precise 'correction term' for the class of the hypersurface of which $Y$ is the singular scheme. The precise statement is

Theorem I.5. Let $X$ be a hypersurface in a nonsingular variety $M$, let $Y$ be its singular scheme, and let $\mathcal{L}=\mathcal{O}(X)$. Then $c_{S M}(X)=c_{*}(X)$, where

$$
c_{*}(X)=c(T M) \cap s(X, M)+c(\mathcal{L})^{\operatorname{dim} X} \cap\left(\mu_{\mathcal{L}}(Y)^{\vee} \otimes_{M} \mathcal{L}\right) .
$$

This statement is equivalent to Theorem I.1-4; showing this amounts to showing that

$$
c(\mathcal{L})^{\operatorname{dim} X} \cap\left(\mu_{\mathcal{L}}(Y) \otimes_{M} \mathcal{L}\right)=c(T M) \cap s(X \backslash Y, M) .
$$

This is a good exercise in the notations of $\S 1.4$, and we leave it to the reader.

The full proof of Theorem I.1-5 occupies $\S \S 2$ and 3: $\S 2$ reduces it to showing that the class $c_{*}$ introduced in this section satisfies a simple blow-up formula ((3) in $\left.\S 2\right)$, and $\S 3$ proves this formula. In the next subsection we summarize an alternative (and shorter) approach to a proof of the main theorem.

1.6. Proof by library search. In principle, the main theorem should follow directly from the extensive current literature on singularities. We include here a sketch of an argument along these lines, pointing to a proof of the theorem as stated in $§ 1.3$. While we are not attempting here to fill in the details involved in proving the key step (the Fact below), the expert will probably find this sketch more insightful than the rather long-winded argument of $\S \S 2$ and 3. On the other hand, the longer argument gives full details, works over any algebraically closed field of characteristic 0 , and puts fewer demands on the background of the reader.

Working in the analytic category over $\mathbb{C}$, let $\mathcal{S}=\left\{S_{\alpha}\right\}$ be a Whitney stratification of the hypersurface $X$ in $M$, and denote by $d_{\alpha}$ the codimension of $S_{\alpha}$ in $X$. Bounded complexes of sheaves $\mathcal{F}^{\bullet}$ on $X$, constructible with respect to $\mathcal{S}$, have a characteristic cycle in $T^{*} M$,

$$
\operatorname{Ch}\left(\mathcal{F}^{\bullet}\right)=\sum_{\alpha} m_{\alpha} \overline{N_{S_{\alpha}}^{*} M}
$$


a combination of the cycles of the closures of the conormal bundles to $S_{\alpha}$ in $M$ (see for example [Massey], p. 356). We view the projectivization $\mathbb{P C h}\left(\mathcal{F}^{\bullet}\right)$ as a cycle in $\mathbb{P}\left(\mathcal{P}_{M}^{1} \mathcal{L}\right)$, via

$$
\mathbb{P}\left(T^{*} M\right) \cong \mathbb{P}\left(T^{*} M \otimes \mathcal{L}\right) \subset \mathbb{P}\left(\mathcal{P}_{M}^{1} \mathcal{L}\right) .
$$

Assume now that the Euler characteristic $\chi\left(\mathcal{F}_{p}^{\bullet}\right)$ of the stalks of $\mathcal{F}^{\bullet}$ is the characteristic function $1_{X}$ of $X$. Then a particular case of Theorem 2 in [BDK] yields

$$
1_{X}=\sum_{\alpha}(-1)^{d_{\alpha}} m_{\alpha} \mathrm{Eu}_{\overline{S_{\alpha}}}
$$

where $\mathrm{Eu}_{\overline{S_{\alpha}}}$ denotes the local Euler obstruction of $\overline{S_{\alpha}}$. Applying MacPherson's natural transformation gives

$$
c_{\mathrm{SM}}(X)=\sum_{\alpha}(-1)^{d_{\alpha}} m_{\alpha} c_{M}\left(\overline{S_{\alpha}}\right) ;
$$

arguing as in Lemma I.2, we can write

$$
c_{M}\left(\overline{S_{\alpha}}\right)=(-1)^{d_{\alpha}} c(T M) \cap \pi_{*}\left(\frac{\left[\mathbb{P} \overline{N_{S_{\alpha}}^{*} M}\right]}{1+X-\mathcal{Y}}\right)
$$

(where, as in $\S 1.3, \pi$ is the projection from $\mathcal{P}_{M}^{1} \mathcal{L}$, and $\mathcal{Y}$ denotes the exceptional divisor of the blow-up of $M$ along the singular subscheme of $X$ ) and therefore

$$
\begin{aligned}
c_{\mathrm{SM}}(X) & =c(T M) \cap \pi_{*} \frac{\sum_{\alpha} m_{\alpha}\left[\mathbb{P} \overline{N_{S_{\alpha}}^{*} M}\right]}{1+X-\mathcal{Y}} \\
& =c(T M) \cap \pi_{*}\left(\frac{\mathbb{P C h}(\mathcal{F} \bullet)}{1+X-\mathcal{Y}}\right) .
\end{aligned}
$$

Comparing with the statement of Theorem I.3, we see that in order to prove the main theorem, it would suffice to show

Fact. For any complex $\mathcal{F}^{\bullet}$ as above,

$$
\operatorname{PCh}\left(\mathcal{F}^{\bullet}\right)=[X]-[\mathcal{Y}]
$$

This is where we leave the expert. This statement looks reasonable set-theoretically, for example by Theorem 3.3 in [Lê-Mebkhout]. It has been suggested to us that the necessary multiplicity computation can be extracted from results in [Parusiński2], but this does not seem immediate. ${ }^{1}$

In a more general set-up, formulas for characteristic cycles can be found in $[\mathrm{BMM}]$. Excellent accounts on the beautiful circle of ideas surrounding characteristic cycles and index formulas are [Sabbah], [Ginsburg], and [Massey].

\section{THE PROOF: PRELIMINARIES}

In this section we set up our strategy for a more elementary proof of the main theorem, by reducing it to the proof of one property (property (3) below) describing the behavior of $c_{*}$ under blow-ups along nonsingular subvarieties. Section 3 is devoted to the proof of (3).

We will need here neither the full functorial picture summarized in the beginning of $\S 1$ nor the details of MacPherson's construction. We will only consider the following three properties of a class $c_{*}$ :

${ }^{1}$ Added in proof: This computation is in fact carried out in a recent preprint by A. Parusiński and P. Pragacz. 
1. If $X$ is a hypersurface, and the support $X_{\text {red }}$ of $X$ is nonsingular, then

$$
c_{*}(X)=c\left(T X_{\text {red }}\right) \cap\left[X_{\text {red }}\right] .
$$

2. Normal crossings: If $X=X_{1} \cup X_{2}$, where $X, X_{2}$ are divisors with normal crossings in a nonsingular ambient variety $M$, and $X_{1}$ is a nonsingular hypersurface of $M$, then

$$
c_{*}(X)=c_{*}\left(X_{1}\right)+c_{*}\left(X_{2}\right)-c_{*}\left(X_{1} \cap X_{2}\right)
$$

(note: we say that a divisor has normal crossings if its support does).

3. Blow-up: If $X$ is a hypersurface of a nonsingular variety $M, Z \subset X \subset M$ is a nonsingular subvariety of codimension $d$ in $M, \pi: \widetilde{M} \rightarrow M$ is the blow-up of $M$ along $Z$, and $X^{\prime}$ denotes the (scheme-theoretic) inverse image of $X$ in $\widetilde{M}$, then

$$
\left(\pi_{\mid X^{\prime}}\right)_{*}\left(c_{*}\left(X^{\prime}\right)\right)=c_{*}(X)+(d-1) c_{*}(Z) .
$$

(We often abuse notations and omit obvious push-forwards and pull-backs, as above.) It is easy to see that the Schwartz-MacPherson class satisfies these properties: (2) follows from the analogous relation between characteristic functions (in fact, with no restrictions on what $X_{1}, X_{2}$ may be); for (3), consider the map

$$
f: X^{\prime} \amalg Z \rightarrow X
$$

restricting to $\pi_{\mid X^{\prime}}$ on $X^{\prime}$ and to the inclusion $i$ into $X$ on $Z$, and define the constructible function $\aleph=1_{X^{\prime}}-(d-1) 1_{Z}$ on $X^{\prime} \amalg Z$. Then for $p \in X$

$$
f_{*}(\aleph)(p)=\left(\pi_{\mid X^{\prime}}\right)_{*}\left(1_{X^{\prime}}\right)(p)-(d-1) i_{*}\left(1_{Z}\right)(p)= \begin{cases}1-0=1 & \text { for } p \notin Z, \\ d-(d-1)=1 & \text { for } p \in Z,\end{cases}
$$

since for $p \in Z, \pi^{-1}(p) \cong \mathbb{P}^{d-1}$ has Euler characteristic $d$. This shows that $f_{*}(\aleph)=$ $1_{X}$, and (3) follows for $c_{\mathrm{SM}}$.

It is also clear from embedded resolution of singularities that the class is uniquely determined by (1), (2), (3) for hypersurfaces $X$ of nonsingular varieties $M$. For this, let $M_{s} \rightarrow M_{s-1} \rightarrow \cdots \rightarrow M_{1} \rightarrow M_{0}=M$ be a sequence of blow-ups at nonsingular centers such that the inverse image $X_{s}$ of $X_{0}=X$ is a divisor with nonsingular components and normal crossings. Then (1) and (2) determine the class for $X_{s}$; and, for $i=s, \ldots, 1, X_{i} \rightarrow X_{i-1}$ is a map as in (3), so the value of the class at $X_{i-1}$ is determined by its value at $X_{i}$.

Summarizing: in order to prove the main theorem, it suffices to show that the class $c_{*}(X)$ introduced in the statements of Theorem I in $§ 1$ satisfies properties (1), (2), (3) above.

The rest of this section is devoted to the proof of (1) and (2) for this class. Property (3) is technically more demanding, and we will devote the entire $\S 3$ to its proof.

2.1. $c_{*}(X)=c_{*}\left(X_{\text {red }}\right)$. Here we prove that under good hypotheses the hypersurface may be assumed to be reduced. Note: it will be a consequence of the main theorem that in fact this holds for all hypersurfaces, but we do not know how to prove this directly in general. The following lemma establishes (1) above, and simplifies the work required to prove (2). 
The context of the lemma is as follows: we want to show that if the components of a hypersurface are sufficiently transversal, then the class of their union is independent of the multiplicity with which the components appear.

Lemma II.1. Let $X_{1}, X_{2}$ be two hypersurfaces in a nonsingular variety $M$, and assume that at every point of $M$ there are local parameters $x_{1}, \ldots, x_{n}$ such that $X_{1}$ has equation $x_{1}=0$, and $X_{2}$ has equation $f\left(x_{2}, \ldots, x_{n}\right)=0$. Also denote by $X_{(m)}$ the hypersurface locally defined by the ideal $\left(x_{1}^{m} f\right)$. Then

$$
c_{*}\left(X_{(m)}\right)=c_{*}\left(X_{(1)}\right) \quad \text { for all } m \geq 1 .
$$

Proof. The jacobian ideal of $X_{(m)}$ is given locally by

$$
x_{1}^{m-1}\left(f, x_{1} \frac{\partial F}{\partial x_{i}}\right)_{i \geq 2} ;
$$

that is, it consists of the $(m-1)$-multiple of $X_{1}$ and of a residual $R$ independent of $m$. But then

$$
B \ell_{Y_{(m)}} M \cong B \ell_{R} M \stackrel{\pi}{\rightarrow} M
$$

is independent of $m$, while the exceptional divisor in $B \ell_{Y_{(m)}}$ is

$$
\mathcal{Y}_{(m)}=(m-1) X_{1}+\mathcal{Y},
$$

where $\mathcal{Y}$ denotes the exceptional divisor in $B \ell_{R} M$. Using the expression for $c_{*}$ in Theorem I.3, we find that

$$
\begin{aligned}
c_{*}\left(X_{(m)}\right) & =c(T M) \cap \pi_{*} \frac{\left[X_{(m)}\right]-\left[\mathcal{Y}_{(m)}\right]}{1+X_{(m)}-\mathcal{Y}_{(m)}} \\
& =c(T M) \cap \pi_{*} \frac{\left(m\left[X_{1}\right]+\left[X_{2}\right]\right)-\left((m-1)\left[X_{1}\right]+[\mathcal{Y}]\right)}{1+\left(m X_{1}+X_{2}\right)-\left((m-1) X_{1}+\mathcal{Y}\right)} \\
& =c(T M) \cap \pi_{*} \frac{\left[X_{1}\right]+\left[X_{2}\right]-[\mathcal{Y}]}{1+X_{1}+X_{2}-\mathcal{Y}}
\end{aligned}
$$

is also independent of $m \geq 1$, as needed.

Lemma II.1 implies (1):

Corollary II.1. If the support $X_{\text {red }}$ of $X$ is nonsingular, then

$$
c_{*}(X)=c\left(T X_{\text {red }}\right) \cap\left[X_{\text {red }}\right] .
$$

Proof. Taking $X_{2}=\emptyset$ in Lemma II.1 yields $c_{*}(X)=c_{*}\left(X_{\text {red }}\right)$, so we may assume $X$ is reduced and nonsingular. Then its singular scheme is $Y=\emptyset$, so $s(X \backslash Y, M)=$ $s(X, M)$. Finally, $s(X, M)=c\left(N_{X} M\right)^{-1} \cap[X]$ (the inverse Chern class of the normal bundle of $X)$, so

$$
c_{*}(X)=c(T M) c\left(N_{X} M\right)^{-1} \cap[X]=c(T X) \cap[X] .
$$

By a divisor with normal crossings we mean a union of smooth distinct hypersurfaces $X_{1} \cup \cdots \cup X_{r}$ such that at each point of intersection of some of the $X_{i}$, say of $X_{1}, \ldots, X_{k}$, there are local coordinates $\left(x_{1}, \ldots, x_{n}\right)$ for the ambient variety so that $x_{1}=0, \ldots, x_{k}=0$ are equations for $X_{1}, \ldots, X_{k}$ respectively. In fact we must allow the $X_{i}$ 's to come with multiplicity: the plan is to apply resolution of singularities to an arbitrary hypersurface $X \subset M$, and this will produce a nonsingular variety mapping to $M$, in which the (scheme-theoretic) inverse image of 
$X$ is a divisor with normal crossings, whose components will appear with multiplicity. Lemma II.1 implies that at this stage we will be able to discard the extra multiplicity information:

Corollary II.2. If $X$ is a (possibly nonreduced) divisor with normal crossing, then

$$
c_{*}(X)=c_{*}\left(X_{\text {red }}\right) .
$$

Proof. This follows by repeatedly applying Lemma II.1, taking for $X_{1}$ each component of the divisor in turn.

It will follow from the main theorem that in fact Corollary II.2 holds for arbitrary hypersurfaces. Again, we do not know how to prove this more general statement directly.

2.2. Divisors with normal crossings. Here we prove that $c_{*}$ satisfies property (2) above. We first translate (2) into the exact form proved below.

Let $X=X_{1} \cup \cdots \cup X_{r}$ be a divisor with normal crossings. By Corollary II.2, above, in computing $c_{*}(X)$ we may assume $X$ is reduced. As usual, $Y$ denotes the singular scheme of $X$ and $\mathcal{L}=\mathcal{O}(X)$. Also, we write $\mathcal{L}_{i}$ for $\mathcal{O}\left(X_{i}\right)$.

Lemma II.2. In order to prove (2), it suffices to show that

$$
s(Y, M)=\left(\left(1-\frac{c\left(\mathcal{L}^{\vee}\right)}{c\left(\mathcal{L}_{1}^{\vee}\right) \cdots c\left(\mathcal{L}_{r}^{\vee}\right)}\right) \cap[M]\right) \otimes_{M} \mathcal{L} .
$$

Here the reader must interpret the right-hand-side as a class supported in $Y$; that is, obvious cancellations must be performed on the right-hand side. This will be assumed implicitly in the following.

Proof. Assuming $s(Y, M)$ is given by the expression in the statement, we derive

$$
s(Y, M)^{\vee} \otimes_{M} \mathcal{L}=\left(1-\frac{c(\mathcal{L})}{c\left(\mathcal{L}_{1}\right) \cdots c\left(\mathcal{L}_{r}\right)}\right) \cap[M]
$$

and therefore (using the expression for $c_{*}$ given in Theorem I.4, and after simple manipulations)

$$
c_{*}(X)=c(T M) \cdot\left(1-\frac{1}{\left(1+X_{1}\right) \cdots\left(1+X_{r}\right)}\right) \cap[M] .
$$

Thus showing (2) for this class amounts to showing that

$$
\begin{aligned}
c(T M) & \left(1-\frac{1}{\left(1+X_{1}\right) \cdots\left(1+X_{r}\right)}\right) \cap[M] \\
= & c\left(T X_{1}\right) \cap\left[X_{1}\right]+c(T M) \cdot\left(1-\frac{1}{\left(1+X_{2}\right) \cdots\left(1+X_{r}\right)}\right) \cap[M] \\
& -c\left(T X_{1}\right)\left(1-\frac{1}{\left(1+X_{2}\right) \cdots\left(1+X_{r}\right)}\right) \cap\left[X_{1}\right],
\end{aligned}
$$

since $X_{1} \cap\left(X_{2} \cup \cdots \cup X_{r}\right)$ is also a reduced divisor with normal crossings (in $X_{1}$, which is assumed to be nonsingular). Now the right-hand side can be written

$$
c(T M)\left(\frac{X_{1}}{\left(1+X_{1}\right) \cdots\left(1+X_{r}\right)}+1-\frac{1}{\left(1+X_{2}\right) \cdots\left(1+X_{r}\right)}\right) \cap[M],
$$

and this is immediately seen to equal the left-hand-side, as needed. 
In passing we note that since $c_{\mathrm{SM}}$ satisfies (2), the following formula must hold for the Schwartz-MacPherson class of a reduced divisor $X=X_{1} \cup \cdots \cup X_{r} \subset M$ as above:

$$
c_{\mathrm{SM}}(X)=c(T M) \cdot\left(1-\frac{1}{\left(1+X_{1}\right) \cdots\left(1+X_{r}\right)}\right) \cap[M] .
$$

Taking degrees in $(*)$ yields an expression for the Euler characteristic of $X$ equivalent to the Claim in $\S 3$ of [Silvotti]. In fact, $\left({ }^{*}\right)$ shows that if $X$ is a normal crossing divisor as above, $c_{\mathrm{SM}}(X)$ maps to the dual of

$$
\left(c\left(\Omega_{M}\right)-c\left(\Omega_{M}(\log X)\right)\right) \cap[M]
$$

in $A_{*} M$, where $\Omega_{M}(\log X)$ denotes the sheaf of logarithmic 1-forms along $X$. Theorem 3.1 in [Silvotti] (that is, 'Gauss-Bonnet for the complement of a divisor') follows by taking degrees in this expression. that

Returning to the proof of (2) for $c_{*}$, by Lemma II.2 we are reduced to showing

$$
s(Y, M)=\left(\left(1-\frac{c\left(\mathcal{L}^{\vee}\right)}{c\left(\mathcal{L}_{1}^{\vee}\right) \cdots c\left(\mathcal{L}_{r}^{\vee}\right)}\right) \cap[M]\right) \otimes_{M} \mathcal{L}
$$

for $Y$ the singular scheme of a reduced divisor $X=X_{1} \cup \cdots \cup X_{r}$ with nonsingular components and normal crossings.

Proof of (2). As a set, $Y$ is the union of all the $X_{i} \cap X_{j}$ with $i \neq j ; X$ has multiplicity $k$ along the intersection of $k$ components, $X_{I}=X_{i_{1}} \cap \cdots \cap X_{i_{k}}(k=|I|)$, provided that this is nonempty. We will work by induction on the number $N$ of nonempty such intersections $X_{I},|I| \geq 2$.

The statement is clear if this number is 0 , that is if $Y$ is empty: $c\left(\mathcal{L}_{1}^{\vee} \otimes \cdots \otimes \mathcal{L}_{r}^{\vee}\right)=$ $c\left(\mathcal{L}_{1}^{\vee}\right) \cdots c\left(\mathcal{L}_{r}^{\vee}\right)$ if the $X_{i}$ 's do not intersect. Assume then $Y \neq \emptyset$, and consider an $X_{I}$ of minimal dimension, say $Z=X_{1} \cap \cdots \cap X_{k}$. Locally along $Z, X_{i}$ has the equation $x_{i}=0$ (for $i \leq k$ ), where the $x_{i}$ 's are part of a system of parameters; so the hypersurface is $x_{1} \cdots x_{k}=0$ along $Z$, and $Z$ has (local) ideal $\left(x_{1}, \ldots, x_{k}\right)$; along $Z, Y$ has ideal

$$
\left(\frac{x_{1} \cdots x_{k}}{x_{1}}, \ldots, \frac{x_{1} \cdots x_{k}}{x_{k}}\right)
$$

Note that if some other hypersurface of the lot came in at some point of $Z$ not covered by the above chart, this would determine a smaller nonempty intersection, against the minimality of $Z$. In other words, $X_{i} \cap Z=\emptyset$ for $i>k$.

Now blow-up $M$ along $Z$; with a suitable choice of coordinates $\tilde{x}_{i}$ in the blow-up, we can write the blow-up map as

$$
\left\{\begin{array}{c}
x_{1}=\tilde{x}_{1} \\
x_{2}=\tilde{x}_{1} \tilde{x}_{2}, \\
\cdots \\
x_{k}=\tilde{x}_{1} \tilde{x}_{k}
\end{array}\right.
$$

and the inverse image of $Y$ has ideal

$$
\left(\frac{\tilde{x}_{1}^{k} \cdots \tilde{x}_{k}}{\tilde{x}_{1}}, \frac{\tilde{x}_{1}^{k} \cdots \tilde{x}_{k}}{\tilde{x}_{1} \tilde{x}_{2}}, \ldots, \frac{\tilde{x}_{1}^{k} \cdots \tilde{x}_{k}}{\tilde{x}_{1} \tilde{x}_{k}}\right)=\tilde{x}_{1}^{k-1}\left(\frac{\tilde{x}_{2} \cdots \tilde{x}_{k}}{\tilde{x}_{2}}, \ldots, \frac{\tilde{x}_{2} \cdots \tilde{x}_{k}}{\tilde{x}_{k}}\right)
$$

in this chart. Now this says that the residual of $(k-1)$ times the exceptional divisor in the inverse image of $Y$ is (in this chart) the singular scheme of the proper 
transform of the hypersurface. This must in fact hold globally on $Z$, as the behavior on the other charts is identical to the one shown above.

Now the key is that the proper transform of the hypersurface is again a divisor with normal crossing, but for which the number $N$ considered above is one less than for the original hypersurface; therefore by induction we know the Segre class of its singular scheme from Lemma II.2:

$$
1-\frac{1-\left(X_{1}-E\right)-\cdots-\left(X_{k}-E\right)-X_{k+1}-\cdots-X_{r}}{\left(1-X_{1}+E\right) \ldots\left(1-X_{k}+E\right)\left(1-X_{k+1}\right) \ldots\left(1-X_{r}\right)} \otimes \mathcal{O}\left(X_{1}+\cdots+X_{r}-k E\right),
$$

where $E$ is the class of the exceptional divisor.

Using Proposition 3 from [Aluffi2] to include the $(k-1)$-multiple $E$, and using the birational invariance of Segre classes, we get that $s(Y, M)$ is the push-forward to $M$ of

$$
\begin{aligned}
& \frac{(k-1) E}{(1+(k-1) E)}+\frac{1}{1+(k-1) E} \\
& \cdot\left(1-\frac{1-X_{1}-\cdots-X_{r}+k E}{\left(1-X_{1}+E\right) \ldots\left(1-X_{k}+E\right)\left(1-X_{k+1}\right) \ldots\left(1-X_{r}\right)} \otimes \mathcal{O}\left(X_{1}+\cdots+X_{r}-E\right)\right) ;
\end{aligned}
$$

that is,

$$
\begin{aligned}
& 1-\frac{1}{1+(k-1) E} \\
& \cdot\left(\frac{1-X_{1}-\cdots-X_{r}+k E}{\left(1-X_{1}+E\right) \ldots\left(1-X_{k}+E\right)\left(1-X_{k+1}\right) \ldots\left(1-X_{r}\right)} \otimes \mathcal{O}\left(X_{1}+\cdots+X_{r}-E\right)\right),
\end{aligned}
$$

and, using [Aluffi2], §2, this is manipulated into

$$
\begin{aligned}
& 1-\left(\frac{1-X_{1}-\cdots-X_{r}+E}{1-X_{1}-\cdots-X_{r}+k E}\right. \\
& \left.\cdot \frac{1-X_{1}-\cdots-X_{r}+k E}{\left(1-X_{1}+E\right) \ldots\left(1-X_{k}+E\right)\left(1-X_{k+1}\right) \ldots\left(1-X_{r}\right)} \otimes \mathcal{O}\left(X_{1}+\cdots+X_{r}-E\right)\right) \\
= & 1-\left(\frac{1-X_{1}-\cdots-X_{r}+E}{\left(1-X_{1}+E\right) \cdots\left(1-X_{k}+E\right)\left(1-X_{k+1}\right) \ldots\left(1-X_{r}\right)} \otimes \mathcal{O}\left(X_{1}+\cdots+X_{r}-E\right)\right) \\
= & 1-\left(\frac{\left(1-X_{1}-\cdots-X_{r}\right)}{\left(1-X_{1}\right) \ldots\left(1-X_{k}\right)} \cdot \frac{(1-E)^{r-1}}{\left(1-X_{k+1}-E\right) \ldots\left(1-X_{r}-E\right)} \otimes \mathcal{O}\left(X_{1}+\cdots+X_{r}\right)\right) .
\end{aligned}
$$

Now we claim that

$$
\frac{(1-E)^{r-1}}{\left(1-X_{k+1}-E\right) \ldots\left(1-X_{r}-E\right)}
$$

pushes forward to

$$
\frac{1}{\left(1-X_{k+1}\right) \ldots\left(1-X_{r}\right)}
$$

Indeed, any term involving both $E$ and some of the $X_{i}$ 's, $i>k$, is necessarily 0 since these $X_{i}$ 's do not meet $Z$; so (*) equals

$$
(1-E)^{k-1}-1+\frac{1}{\left(1-X_{k+1}\right) \ldots\left(1-X_{r}\right)},
$$

and all powers $E^{i}$ with $0<i<k$ push forward to 0 because $Z$ has codimension $k$. So $\left({ }^{*}\right)$ is all that survives the push-forward.

In conclusion, this shows that $s(Y, M)$ equals

$$
[M]-\frac{\left([M]-\left[X_{1}\right]-\cdots-\left[X_{r}\right]\right)}{\left(1-X_{1}\right) \ldots\left(1-X_{r}\right)} \otimes_{M} \mathcal{O}\left(X_{1}+\cdots+X_{r}\right),
$$

completing the induction step. 
This concludes the proof that the class $c_{*}$ of $\S \S 1$ and 2 satisfies properties (1) and (2) stated at the beginning of this section (and it follows that $c_{*}$ and the Schwartz-MacPherson class coincide for hypersurfaces with normal crossing).

$\S 3$ is devoted to the proof of (3), thereby concluding the proof of Theorem I. This will be the most delicate ingredient in the proof of the main theorem.

\section{BEHAVIOR UNDER BLOW-UPS}

The last ingredient in the elementary proof of the main theorem is the proof of (3) from $\S 2$. We will obtain this by transforming (3) into equivalent and more basic assertions, which however will require more and more notations to be stated. In the end, (3) will follow by an explicit computation of a 'cycle at infinity' ( $Z_{\infty}$ in $\S \S 3.4-3.8)$ arising in a graph construction.

We first reproduce the notations used so far, and the statement of (3) given in $\S 2$. Let $X$ be a hypersurface of a nonsingular variety $M$, and let $Z \subset X \subset M$ be a nonsingular subvariety of codimension $d$ in $M . \widetilde{M} \stackrel{\pi}{\rightarrow} M$ will be the blow-up of $M$ along $Z, E$ will denote the exceptional divisor of this blow-up, and $X^{\prime}$ the scheme-theoretic inverse image of $X$ in $\widetilde{M} ; \mathcal{L}$ will be the line bundle of $X$ (hence its pull-back, also denoted $\mathcal{L}$, is the line bundle of $X^{\prime}$ ), and $Y, Y^{\prime}$ will respectively denote the singular subschemes of $X, X^{\prime}$. Then (3) states that

$$
\pi_{*}\left(c_{*}\left(X^{\prime}\right)\right)=c_{*}(X)+(d-1) c_{*}(Z)
$$

in $A_{*} X$. (Note: in this section especially we will often indulge in severe notational abuses, of which this formula is a good example. To interpret this formula correctly, the reader is expected to restrict $\pi$ to $X^{\prime}$ before using it to push forward $c_{*}\left(X^{\prime}\right)$; and to push forward $c_{*}(Z)$ from $A_{*} Z$ to $A_{*} X$. While this will make some of our statements slightly imprecise, employing full notations would often make them quite unreadable; we opt for the first alternative.)

3.1. (3) in terms of classes in $\mathbb{P P}_{M}^{1} \mathcal{L}, \mathbb{P} \mathcal{P} \frac{1}{M} \mathcal{L}$. Here we translate (3) by using the form of $c_{*}$ given in Theorem I.3.

We will denote by $\mathcal{P}_{M}^{1} \mathcal{L}, \mathcal{P} \frac{1}{M} \mathcal{L}$ respectively the bundles of principal parts of $\mathcal{L}$ over $M, \widetilde{M}$. The section $F$ of $\mathcal{L}$ over $M, M^{\prime}$ defining $X, X^{\prime}$ resp. determine sections

$$
\widetilde{M} \rightarrow \pi^{*} \mathcal{P}_{M}^{1} \mathcal{L}, \quad \widetilde{M} \rightarrow \mathcal{P}_{\widetilde{M}}^{1} \mathcal{L},
$$

which projectivize to rational maps

$$
\widetilde{M} \rightarrow \mathbb{P} \pi^{*} \mathcal{P}_{M}^{1} \mathcal{L}, \quad \widetilde{M} \rightarrow \mathcal{P} \frac{1}{\widetilde{L}} \mathcal{L}
$$

The closures of the images of these maps are the blow-ups $B \ell_{\pi^{-1} Y} \widetilde{M}, B \ell_{Y^{\prime}} \widetilde{M}$ respectively (this follows from staring at local descriptions for the sections; cf. $\S 1.3$ ).

This is the first instance in which we perform two parallel constructions: one on the $\pi^{*} \mathcal{P}_{M}^{1} \mathcal{L}$ side, the other on the $\mathcal{P} \frac{1}{\widetilde{L}} \mathcal{L}$ side. As a rule, we will put subscripts $M$, $\widetilde{M}$ on corresponding objects on the two sides, to keep track of which side they belong to: we start this convention by naming the universal subbundles in $\mathbb{P} \pi^{*} \mathcal{P}_{M}^{1} \mathcal{L}$, $\mathbb{P} \mathcal{P} \frac{1}{\widetilde{L}} \mathcal{L}$ respectively $\mathcal{O}_{M}(-1), \mathcal{O}_{\widetilde{M}}(-1)$. Similarly, $\mathcal{Y}_{M}, \mathcal{Y}_{\widetilde{M}}$ will denote respectively the exceptional divisors in $B \ell_{\pi^{-1} Y} \widetilde{M}, B \ell_{Y^{\prime}} \widetilde{M}$; note that $\mathcal{O}\left(\mathcal{Y}_{M}\right), \mathcal{O}\left(\mathcal{Y}_{\widetilde{M}}\right)$ are respectively the restriction of $\mathcal{O}_{M}(-1), \mathcal{O}_{\widetilde{M}}(-1)$ to the blow-ups. Also, $p_{M}, p_{\widetilde{M}}$ will denote respectively the bundle maps on $\mathbb{P} \pi^{*} \mathcal{P}_{M}^{1} \mathcal{L}, \mathbb{P} \mathcal{P} \frac{1}{M} \mathcal{L}$. Finally, the reader is 
warned that the $\pi^{*}$ employed so far will soon be dropped (as is allowed by various functorialities of pull-backs).

Claim III.1. In order to prove (3), it suffices to show that

$$
\pi_{*} p_{M_{*}}\left(c\left(\frac{\mathcal{P}_{M}^{1} \mathcal{L}}{\mathcal{O}_{M}(-1)}\right) \cap\left[B \ell_{\pi^{-1} Y} \widetilde{M}\right]\right)=\pi_{*} p_{\widetilde{M} *}\left(c\left(\frac{\mathcal{P}_{\widetilde{M}}^{1} \mathcal{L}}{\mathcal{O}_{\widetilde{M}}(-1)}\right) \cap\left[B \ell_{Y^{\prime}}, \widetilde{M}\right]\right) .
$$

Proof. Writing $c_{*}$ as in $\S 1.3$, and with the above notations,

$$
\begin{gathered}
c_{*}(X)=\pi_{*}\left(c(T M) \cap p_{M *}\left(\frac{[X]-\left[\mathcal{Y}_{M}\right]}{1+X-\mathcal{Y}_{M}}\right)\right), \\
c_{*}\left(X^{\prime}\right)=c(T \widetilde{M}) \cap p_{\widetilde{M} *}\left(\frac{\left[X^{\prime}\right]-\left[\mathcal{Y}_{\widetilde{M}}\right]}{1+X^{\prime}-\mathcal{Y}_{\widetilde{M}}}\right) .
\end{gathered}
$$

Now

$$
\begin{aligned}
& \pi_{*} p_{M_{*}}\left(c\left(\frac{\mathcal{P}_{M}^{1} \mathcal{L}}{\mathcal{O}_{M}(-1)}\right) \cap\left[B \ell_{\pi^{-1} Y} \widetilde{M}\right]\right)-\pi_{*} p_{\widetilde{M} *}\left(c\left(\frac{\mathcal{P}_{\widetilde{M}}^{1} \mathcal{L}}{\mathcal{O}_{\widetilde{M}}(-1)}\right) \cap\left[B \ell_{Y^{\prime}} \widetilde{M}\right]\right) \\
& =\pi_{*}\left(p_{M_{*}}\left(\frac{c\left(T^{*} M \otimes \mathcal{L}\right) c(\mathcal{L})}{1+\mathcal{Y}_{M}} \cap\left[B \ell_{\pi^{-1} Y} \widetilde{M}\right]\right)-p_{\widetilde{M} *}\left(\frac{c\left(T^{*} \widetilde{M} \otimes \mathcal{L}\right) c(\mathcal{L})}{1+\mathcal{Y}_{\widetilde{M}}} \cap\left[B \ell_{Y^{\prime}} \widetilde{M}\right]\right)\right) \\
& =c(\mathcal{L})^{n+1} \pi_{*}\left(p_{M_{*}}\left(\frac{c\left(T^{*} M\right)}{1-X+\mathcal{Y}_{M}} \cap\left[B \ell_{\pi^{-1} Y} \widetilde{M}\right]\right)-p_{\widetilde{M} *}\left(\frac{c\left(T^{*} \widetilde{M}\right)}{1-X^{\prime}+\mathcal{Y}_{\widetilde{M}}} \cap\left[B \ell_{Y^{\prime}} \widetilde{M}\right]\right)\right) \\
& \quad \otimes \mathcal{L}
\end{aligned}
$$

(notations as in $\S 1.4$, and properties of the same from [Aluffi2]). Thus the equality in the statement is equivalent to

$$
\pi_{*}\left(p_{M *}\left(\frac{c\left(T^{*} M\right)}{1-X+\mathcal{Y}_{M}} \cap\left[B \ell_{\pi^{-1} Y} \widetilde{M}\right]\right)-p_{\widetilde{M} *}\left(\frac{c\left(T^{*} \widetilde{M}\right)}{1-X^{\prime}+\mathcal{Y}_{\widetilde{M}}} \cap\left[B \ell_{Y^{\prime}} \widetilde{M}\right]\right)\right)=0
$$

Taking duals, this is equivalent to

$$
\pi_{*}\left(p_{M *}\left(\frac{c(T M)}{1+X-\mathcal{Y}_{M}} \cap\left[B \ell_{\pi^{-1} Y} \widetilde{M}\right]\right)-p_{\widetilde{M} *}\left(\frac{c(T \widetilde{M})}{1+X^{\prime}-\mathcal{Y}_{\widetilde{M}}} \cap\left[B \ell_{Y^{\prime}} \widetilde{M}\right]\right)\right)=0,
$$

that is, to

$$
\begin{aligned}
\pi_{*} p_{M *}(c(T M)(1- & \left.\left.\frac{X-\mathcal{Y}_{M}}{1+X-\mathcal{Y}_{M}}\right) \cap\left[B \ell_{\pi^{-1} Y} \widetilde{M}\right]\right) \\
& -\pi_{*} p_{\widetilde{M}}\left(c(T \widetilde{M})\left(1-\frac{X^{\prime}-\mathcal{Y}_{\widetilde{M}}}{1+X^{\prime}-\mathcal{Y}_{\widetilde{M}}}\right) \cap\left[B \ell_{Y^{\prime}} \widetilde{M}\right]\right)=0,
\end{aligned}
$$

or, using the expressions given above for $c_{*}$, to

$$
\pi_{*} p_{M_{*}}\left(c(T M) \cap\left[B \ell_{\pi^{-1} Y} \widetilde{M}\right]\right)-c_{*}(X)-\pi_{*} p_{\widetilde{M} *}\left(c(T \widetilde{M}) \cap\left[B \ell_{Y^{\prime}} \widetilde{M}\right]\right)+\pi_{*} c_{*}\left(X^{\prime}\right)=0,
$$

and finally, using the projection formula, to

$$
\pi_{*} c_{*}\left(X^{\prime}\right)=c(X)+\pi_{*}((c(T \widetilde{M})-c(T M)) \cap[\widetilde{M}]) .
$$


Now

$$
\pi_{*}(c(T \widetilde{M}) \cap[\widetilde{M}])-c(T M) \cap[M]=(d-1) c(T Z) \cap[Z]:
$$

in characteristic 0 this is immediate from the functoriality of Schwartz-MacPherson classes (in fact it holds in arbitrary characteristic, as may be easily checked using Theorem 15.4 in [Fulton]). Therefore the equality in the statement is equivalent to

$$
\pi_{*} c_{*}\left(X^{\prime}\right)=c(X)+(d-1) c(T Z) \cap[Z]
$$

which is precisely (3), as needed.

3.2. (3) in terms of classes in $\mathbb{P}\left(\mathcal{P}_{M}^{1} \mathcal{L} \oplus \mathcal{P} \frac{1}{M} \mathcal{L}\right)$ over $\widetilde{M}$. Before attacking the equality stated in Claim III.1, we need another notational layer to put both sides in the same place. The general plan is to show that they are equal by realizing them as cycles arising in a graph construction ([MacPherson], [BFM], or [Fulton], $\S 18.1)$. The natural place to look for something of the sort is

$$
\mathbb{P}\left(\mathcal{P}_{M}^{1} \mathcal{L} \oplus \mathcal{P}_{\widetilde{M}}^{1} \mathcal{L}\right)
$$

There are two natural embeddings:

$$
\mathbb{P}\left(\mathcal{P}_{M}^{1} \mathcal{L}\right) \hookrightarrow \mathbb{P}\left(\mathcal{P}_{M}^{1} \mathcal{L} \oplus \mathcal{P}_{\widetilde{M}}^{1} \mathcal{L}\right), \quad \mathbb{P}\left(\mathcal{P}_{\widetilde{M}}^{1} \mathcal{L}\right) \hookrightarrow \mathbb{P}\left(\mathcal{P}_{M}^{1} \mathcal{L} \oplus \mathcal{P}_{\widetilde{M}}^{1} \mathcal{L}\right)
$$

as 'first', resp. 'second' factor. These are the centers of two families of central projections

$$
\mathbb{P}\left(\mathcal{P}_{M}^{1} \mathcal{L} \oplus \mathcal{P}_{\widetilde{M}}^{1} \mathcal{L}\right)_{--\rightarrow}^{\rho_{\widetilde{M}}} \mathbb{P}\left(\mathcal{P}_{\widetilde{M}}^{1} \mathcal{L}\right), \quad \mathbb{P}\left(\mathcal{P}_{M}^{1} \mathcal{L} \oplus \mathcal{P}_{\widetilde{M}}^{1} \mathcal{L}\right)_{\stackrel{\rho}{M}}^{\rightarrow} \mathbb{P}\left(\mathcal{P}_{M}^{1} \mathcal{L}\right)
$$

respectively. Also, the rational maps from $\widetilde{M}$ to the bundles (considered above) determine two embeddings

$$
\begin{gathered}
B \ell_{\pi^{-1} Y} \widetilde{M} \hookrightarrow \mathbb{P}\left(\mathcal{P}_{M}^{1} \mathcal{L}\right) \hookrightarrow \mathbb{P}\left(\mathcal{P}_{M}^{1} \mathcal{L} \oplus \mathcal{P} \frac{1}{\widetilde{M}} \mathcal{L}\right) \\
B \ell_{Y^{\prime}} \widetilde{M} \hookrightarrow \mathbb{P}\left(\mathcal{P}_{\widetilde{M}}^{1} \mathcal{L}\right) \hookrightarrow \mathbb{P}\left(\mathcal{P}_{M}^{1} \mathcal{L} \oplus \mathcal{P}_{\widetilde{M}}^{1} \mathcal{L}\right)
\end{gathered}
$$

and the cones

$$
G_{M}=\overline{\rho_{M}^{-1}\left(B \ell_{\pi^{-1} Y} \widetilde{M}\right)}, \quad G_{\widetilde{M}}=\overline{\rho_{\widetilde{M}}^{-1}\left(B \ell_{Y^{\prime}} \widetilde{M}\right)}
$$

Also denote by $\mathcal{O}(-1)$ the tautological subbundle of $\mathbb{P}\left(\mathcal{P}_{M}^{1} \mathcal{L} \oplus \mathcal{P} \frac{1}{\widetilde{L}} \mathcal{L}\right)$, and note that $\mathcal{O}(-1)$ restricts to $\mathcal{O}_{M}(-1), \mathcal{O}_{\widetilde{M}}(-1)$ on the two factors, and that

$$
\begin{aligned}
& N_{\mathbb{P}\left(\mathcal{P}_{M}^{1} \mathcal{L}\right)} \mathbb{P}\left(\mathcal{P}_{M}^{1} \mathcal{L} \oplus \mathcal{P}_{\widetilde{M}}^{1} \mathcal{L}\right)=\mathcal{P} \frac{1}{\mathcal{M}^{\prime}} \mathcal{L} \otimes \mathcal{O}(1) \\
& N_{\mathbb{P}\left(\mathcal{P}_{\overparen{M}}^{1} \mathcal{L} \mathcal{L}\right.} \mathbb{P}\left(\mathcal{P}_{M}^{1} \mathcal{L} \oplus \mathcal{P}_{\widetilde{M}}^{1} \mathcal{L}\right)=\mathcal{P}_{M}^{1} \mathcal{L} \otimes \mathcal{O}(1)
\end{aligned}
$$


(as seen with the aid of standard Euler sequences). Finally, $p$ will denote the bundle map to $\widetilde{M}$. Here are some of the notations in a diagram:

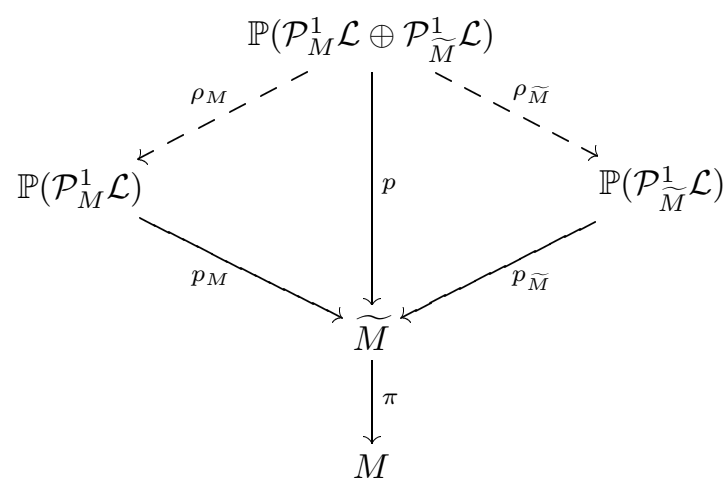

Now we are ready for the new reformulation of what we have to prove:

Claim III.2. In order to prove (3), it suffices to show that

$$
c\left(\frac{\mathcal{P}_{M}^{1} \mathcal{L} \oplus \mathcal{P}_{\widetilde{M}}^{1} \mathcal{L}}{\mathcal{O}(-1)}\right) \cap\left(\left[G_{\widetilde{M}}\right]-\left[G_{M}\right]\right)
$$

pushes forward to 0 in $M$.

Proof. This follows immediately from Claim III.1 and the following lemma:

\section{Lemma III.1.}

$$
\begin{aligned}
& p_{*}\left(c\left(\frac{\mathcal{P}_{M}^{1} \mathcal{L} \oplus \mathcal{P}_{\widetilde{M}}^{1} \mathcal{L}}{\mathcal{O}(-1)}\right) \cap\left[G_{M}\right]\right)=p_{M_{*}}\left(c\left(\frac{\mathcal{P}_{M}^{1} \mathcal{L}}{\mathcal{O}_{M}(-1)}\right) \cap\left[B \ell_{\pi^{-1} Y} \widetilde{M}\right]\right), \\
& p_{*}\left(c\left(\frac{\mathcal{P}_{M}^{1} \mathcal{L} \oplus \mathcal{P} \frac{\widetilde{M}}{\mathcal{L}} \mathcal{L}}{\mathcal{O}(-1)}\right) \cap\left[G_{\widetilde{M}}\right]\right)=p_{\widetilde{M} *}\left(c\left(\frac{\mathcal{P}_{\widetilde{M}}^{1} \mathcal{L}}{\mathcal{O}_{\widetilde{M}}(-1)}\right) \cap\left[B \ell_{Y^{\prime}} \widetilde{M}\right]\right) .
\end{aligned}
$$

Proof. We check the first equality; the second is entirely similar.

First, observe that, since $G_{M} \cap \mathbb{P}\left(\mathcal{P}_{M}^{1} \mathcal{L}\right)=B \ell_{\pi^{-1} Y} \widetilde{M}$ in $\mathbb{P}\left(\mathcal{P}_{M}^{1} \mathcal{L} \oplus \mathcal{P}_{\widetilde{M}}^{1} \mathcal{L}\right.$ ) (and this intersection is transversal),

$$
p_{M *}\left(c\left(\frac{\mathcal{P}_{M}^{1} \mathcal{L}}{\mathcal{O}_{M}(-1)}\right) \cap\left[B \ell_{\pi^{-1} Y} \widetilde{M}\right]\right)=p_{*}\left(c\left(\frac{\mathcal{P}_{M}^{1} \mathcal{L}}{\mathcal{O}_{M}(-1)}\right) \cap\left[\mathbb{P}\left(\mathcal{P}_{M}^{1} \mathcal{L}\right)\right] \cdot\left[G_{M}\right]\right) .
$$

Next, $\mathcal{O}(1)$ restricts to $\mathcal{O}_{M}(1)$ on $\mathbb{P}\left(\mathcal{P}_{M}^{1} \mathcal{L}\right)$; denote by $j$ its first Chern class. The normal bundle formula above tells us that (with $n=\operatorname{dim} M$ )

$$
\begin{aligned}
{\left[\mathbb{P}\left(\mathcal{P}_{M}^{1} \mathcal{L}\right)\right] \cdot\left[G_{M}\right] } & =c_{\text {top }}\left(\mathcal{P}_{\widetilde{M}}^{1} \mathcal{L} \otimes \mathcal{O}(1)\right) \cap\left[G_{M}\right] \\
& =\left(j^{n+1}+\cdots+j c_{n}\left(\mathcal{P}_{\widetilde{M}}^{1} \mathcal{L}\right)+c_{n+1}\left(\mathcal{P}_{\widetilde{M}}^{1} \mathcal{L}\right)\right) \cap\left[G_{M}\right] .
\end{aligned}
$$

Therefore

$$
\begin{aligned}
& c\left(\frac{\mathcal{P}_{M}^{1} \mathcal{L}}{\mathcal{O}_{M}(-1)}\right) \cap\left[\mathbb{P}\left(\mathcal{P}_{M}^{1} \mathcal{L}\right)\right] \cdot\left[G_{M}\right] \\
& =c\left(\mathcal{P}_{M}^{1} \mathcal{L}\right)\left(1+j+j^{2}+\ldots\right)\left(j^{n+1}+\cdots+j c_{n}\left(\mathcal{P}_{\widetilde{M}}^{1} \mathcal{L}\right)+c_{n+1}\left(\mathcal{P}_{\widetilde{M}}^{1} \mathcal{L}\right)\right) \cap\left[G_{M}\right] .
\end{aligned}
$$


With the same notations,

$$
\begin{aligned}
& c\left(\frac{\mathcal{P}_{M}^{1} \mathcal{L} \oplus \mathcal{P}_{\widetilde{M}}^{1} \mathcal{L}}{\mathcal{O}(-1)}\right) \cap\left[G_{M}\right] \\
& \quad=c\left(\mathcal{P}_{M}^{1} \mathcal{L}\right)\left(1+j+j^{2}+\ldots\right)\left(1+\cdots+c_{n}\left(\mathcal{P}_{\widetilde{M}}^{1} \mathcal{L}\right)+c_{n+1}\left(\mathcal{P}_{\widetilde{M}}^{1} \mathcal{L}\right)\right) \cap\left[G_{M}\right] .
\end{aligned}
$$

The difference consists of a sum of terms

$$
c\left(\mathcal{P}_{M}^{1} \mathcal{L}\right) j^{i} c_{k}\left(\mathcal{P} \frac{1}{\widetilde{M}} \mathcal{L}\right) \cap\left[G_{M}\right]
$$

with $i+k<n+1$; but $G_{M}$ fibers over its image in $\mathbb{P}\left(\mathcal{P}_{M}^{1} \mathcal{L}\right)$ (via $\rho_{M}$ ) with fibers of dimension $n+1$, so all such terms die already in $\mathbb{P}\left(\mathcal{P}_{M}^{1} \mathcal{L}\right)$; and a fortiori after push-forward to $\widetilde{M}$.

3.3. (3) in terms of classes in $\mathbb{P}\left(\mathcal{P}_{M}^{1} \mathcal{L} \oplus \mathcal{P} \frac{1}{\widetilde{M}} \mathcal{L}\right)$ over $B L$. In order to attack Claim III.2, we have to produce explicitly a class equivalent to $\left[G_{\widetilde{M}}\right]-\left[G_{M}\right]$, and we have to evaluate the intersection product and push-forward stated in Claim III.2. For this, we will pull-back the situation to a variety dominating both $B \ell_{\pi^{-1} Y} \widetilde{M}$ and $B \ell_{Y}, \widetilde{M}$. Consider the natural morphism of bundles

$$
\phi: \quad \mathcal{P}_{M}^{1} \mathcal{L} \rightarrow \mathcal{P}_{\widetilde{M}}^{1} \mathcal{L}
$$

over $\widetilde{M}$, extending the differential $d \pi: T^{*} M \rightarrow T^{*} \widetilde{M}$. (We are omitting here, and we will omit from now on, the pull-back notation $\pi^{*}$ on the sources of these morphisms.) This morphism will play a fundamental role in what follows.

For a start, observe that $\phi$ is also a family of central projections: over a general point of $\widetilde{M}, \phi$ is an isomorphism; over a point of $E$, say corresponding to a direction $u$ normal to $Z, \phi$ collapses forms vanishing along $T Z$ and $u$. Projectivizing, we get a rational map

$$
\psi: \quad \mathbb{P}_{M}^{1} \mathcal{L} \rightarrow \mathbb{P} \mathcal{P} \frac{1}{M} \mathcal{L}
$$

which is resolved by blowing up the family $C$ of centers of the projections (with the reduced structure); equivalently, the blow-up will be the graph $\Gamma$ of $\psi$ in $\mathbb{P}_{M}^{1} \mathcal{L} \times \widetilde{M}$ $\mathbb{P} \mathcal{P} \frac{1}{M} \mathcal{L}$. At the same time, $\psi$ restricts to a rational map

$$
B \ell_{\pi^{-1} Y} \widetilde{M} \rightarrow B \ell_{Y^{\prime}}, \widetilde{M}
$$

which can be resolved by blowing up the source along its intersection with $C$, obtaining a variety $B L$. Equivalently, $B L$ is the graph of this map, which sits in $\Gamma$ :

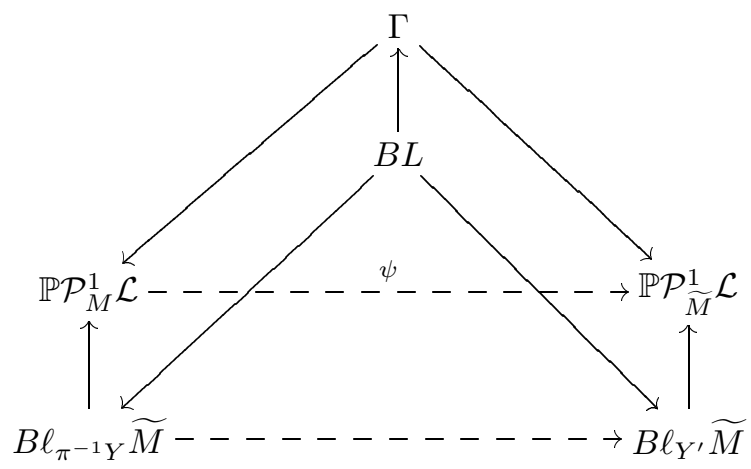


Since $B L$ maps to both $B \ell_{\pi^{-1} Y} \widetilde{M}, B \ell_{Y^{\prime}} \widetilde{M}$, note that on $B L$ we have line bundles (obtained by pulling back) $\mathcal{O}_{M}(-1)=\mathcal{O}\left(\mathcal{Y}_{M}\right), \mathcal{O}_{\widetilde{M}}(-1)=\mathcal{O}\left(\mathcal{Y}_{\widetilde{M}}\right)$.

Now we can pull-back $\mathbb{P}\left(\mathcal{P}_{M}^{1} \mathcal{L} \oplus \mathcal{P}_{\widetilde{M}}^{1} \mathcal{L}\right)$ etc. to $B L$. The advantage of doing so is that the cycles playing the role of $G_{M}, G_{\widetilde{M}}$ have a nicer description: consider the following loci defined over $B L$ :

$$
\begin{aligned}
G_{M} & =\mathbb{P}\left(\mathcal{O}_{M}(-1) \oplus \mathcal{P}_{\widetilde{M}}^{1} \mathcal{L}\right) \subset \mathbb{P}\left(\mathcal{P}_{M}^{1} \mathcal{L} \oplus \mathcal{P} \widetilde{M}^{1} \mathcal{L}\right), \\
G_{\widetilde{M}} & =\mathbb{P}\left(\mathcal{P}_{M}^{1} \mathcal{L} \oplus \mathcal{O}_{\widetilde{M}}(-1)\right) \subset \mathbb{P}\left(\mathcal{P}_{M}^{1} \mathcal{L} \oplus \mathcal{P}_{\widetilde{M}}^{1} \mathcal{L}\right)
\end{aligned}
$$

the reader will verify that these $G_{M}, G_{\widetilde{M}}$ push forward to the loci with the same name over $\widetilde{M}$ (the reason is that $\mathbb{P}\left(\mathcal{O}_{M}(-1)\right) \subset \mathbb{P}\left(\mathcal{P}_{M}^{1} \mathcal{L}\right)$ realizes the embedding of $B \ell_{\pi^{-1} Y} M$ in $\mathbb{P P}_{M}^{1} \mathcal{L}$, etc.).

It follows that we can adopt Claim III.2, taking the bundle and cycles in the statement to live now over $B L$, with the above positions.

3.4. The graph construction. The loci $G_{M}, G_{\widetilde{M}}$ are now projectivizations of rank- $(n+2)$ subbundles of $\mathcal{P}_{M}^{1} \mathcal{L} \oplus \mathcal{P}_{\widetilde{M}}^{1} \mathcal{L}$ over $B L$. It is natural to interpolate them by considering the span of the graph of

$$
\frac{1}{\lambda} \phi: \quad \mathcal{P}_{M}^{1} \mathcal{L} \rightarrow \mathcal{P} \frac{1}{M} \mathcal{L}
$$

and $0 \oplus \mathcal{O}_{\widetilde{M}}(-1)$ in $\mathcal{P}_{M}^{1} \mathcal{L} \oplus \mathcal{P}_{\widetilde{M}}^{1} \mathcal{L}$ : let $G_{\lambda}$ denote this span (thus $G_{\lambda}$ is a rank- $(n+2)$ subbundle of $\mathcal{P}_{M}^{1} \mathcal{L} \oplus \mathcal{P}_{\widetilde{M}}^{1} \mathcal{L}$ for all $\lambda \neq 0$ ).

Notice that as $1 / \lambda \rightarrow 0, G_{\lambda}$ projectivizes to the locus $G_{\widetilde{M}}$ defined above. In fact $G_{\lambda}$ can be realized (for $\lambda \neq 0$ ) as the element in $\operatorname{Grass}_{n+2}\left(\mathcal{P}_{M}^{1} \mathcal{L} \oplus \mathcal{P} \frac{1}{M} \mathcal{L}\right)$ determined by the graph of

$$
\mathcal{P}_{M}^{1} \mathcal{L} \stackrel{\frac{1}{\lambda} \phi}{\longrightarrow} \mathcal{P} \frac{1}{\widetilde{M}} \mathcal{L} \rightarrow \frac{\mathcal{P}_{\widetilde{M}}^{1} \mathcal{L}}{\mathcal{O}_{\widetilde{M}}(-1)}
$$

a point of $\operatorname{Grass}_{n+1}\left(\mathcal{P}_{M}^{1} \mathcal{L} \oplus\left(\mathcal{P}_{\widetilde{M}}^{1} \mathcal{L} / \mathcal{O}_{\widetilde{M}}(-1)\right)\right)$. Over general points of $B L$, the kernel of the composition $\left(^{*}\right)$ is the fiber of $\mathcal{O}_{M}(-1)$; it follows from general considerations about the graph construction ([BFM]; also [Kwieciński], I.7, p. 56) that the flat limit of $G_{\lambda}$ as $1 / \lambda \rightarrow \infty$ will consist of several components, one of which will precisely projectivize to the locus $G_{M}$ defined above. This will also be recovered later on, by a coordinate computation.

Let $Z_{\infty}$ denote the other (that is, distinct from $G_{M}$ ) components of the projectivization of $\lim _{1 / \lambda \mapsto \infty} G_{\lambda}$.

Claim III.3. In order to prove (3), it suffices to show that

$$
c\left(\frac{\mathcal{P}_{M}^{1} \mathcal{L} \oplus \mathcal{P}_{\widetilde{M}}^{1} \mathcal{L}}{\mathcal{O}(-1)}\right) \cap\left[Z_{\infty}\right]
$$

pushes forward to 0 in $M$.

Proof. The construction gives an explicit rational equivalence between $\left[G_{\widetilde{M}}\right]=$ $\left[\lim _{1 / \lambda \mapsto 0} \mathbb{P}\left(G_{\lambda}\right)\right]$ and $\left[\lim _{1 / \lambda \mapsto \infty} \mathbb{P}\left(G_{\lambda}\right)\right]=\left[G_{M}\right]+\left[Z_{\infty}\right]$. 
3.5. Coordinate set-up in $B L$. Fortunately $\lim _{\lambda \mapsto 0} G_{\lambda}$ (and therefore $Z_{\infty}$ ) can be analyzed most explicitly by a coordinate computation; however, this requires studying $B L$ more carefully and introducing if possible yet more notations.

The variety $B L$ contains (inverse images of) divisors $E, \mathcal{Y}_{M}, \mathcal{Y}_{\widetilde{M}}$; in fact we already observed that the line bundles for the latter two are respectively $\mathcal{O}_{M}(-1)$, $\mathcal{O}_{\widetilde{M}}(-1)$. Also, as $B L$ arises by blowing up $B \ell_{\pi^{-1} Y} \widetilde{M}$ along a certain locus (called $C$ in the above), it contains a corresponding exceptional divisor $\mathcal{E}_{M}$. Further, note that the rational map $\psi$ defined above is birational; so we can think of $B L$ as the resolution of indeterminacies of $\psi^{-1}$, which realizes it as a blow-up of $B \ell_{Y^{\prime}} \widetilde{M}$. Denote by $\mathcal{E}_{\widetilde{M}}$ the exceptional divisor of this blow-up. (Note: $\mathcal{E}_{M}, \mathcal{E}_{\widetilde{M}}$ are restrictions of analogous exceptional divisors from $\Gamma$.)

The intersection of $C$ and $B \ell_{\pi^{-1} Y} \widetilde{M}$ can be computed easily. The reader may wish to check that it is the residual to $\mathcal{Y}_{M}$ of the scheme-theoretic inverse image of $Y^{\prime}$ in $B \ell_{\pi^{-1} Y} \widetilde{M}$. Similarly, it is not hard to see that the center of the blow-up of $B \ell_{Y}, \widetilde{M}$ producing $B L$ is the residual to $\mathcal{Y}_{\widetilde{M}}$ in the (scheme theoretic) union of $E$ and the inverse image of $Y$ in $B \ell_{Y}, \widetilde{M}$.

To get a feeling for the situation, the reader may also wish to check that the residual to $Y$ in $Y^{\prime}$ is supported on the intersection of $E$ and the proper transform $\widetilde{X}$ of $X$ in $\widetilde{M}$. In fact, $E \cap Y^{\prime}$ is precisely this intersection, while $E \cap Y$ is supported 'just' on the points at which $\widetilde{X}$ is tangent to the fibers of $E$ over $Z$.

Lemma III.2. With the above notations

$$
\begin{gathered}
\mathcal{E}_{M}+\mathcal{E}_{\widetilde{M}}=E, \\
\mathcal{Y}_{M}+\mathcal{E}_{M}=\mathcal{Y}_{\widetilde{M}},
\end{gathered}
$$

as divisors of $B L$.

Proof. These equalities follow easily from the considerations immediately preceding this statement. For example, the ideal of $\mathcal{E}_{M}$ is the pull-back of the ideal of $C$, hence it is the residual to $\mathcal{Y}_{M}$ in $\mathcal{Y}_{\widetilde{M}}$; this gives the second equality. By the same token, $\mathcal{E}_{\widetilde{M}}$ is the residual to $\mathcal{Y}_{\widetilde{M}}$ in $\mathcal{Y}_{M}+E$; that is,

$$
\mathcal{Y}_{M}+E=\mathcal{Y}_{\widetilde{M}}+\mathcal{E}_{\widetilde{M}},
$$

and the first equality follows.

Lemma III.2 will be used in a moment, when we choose functions on $B L$ to write entries for a matrix whose row-span is $G_{\lambda}$. First, we have to choose local coordinates in $\mathcal{P}_{M}^{1} \mathcal{L}$ and $\mathcal{P} \widetilde{M}^{1} \mathcal{L}$. Choose local parameters $x_{1}, \ldots, x_{n}$ in $M$ and $\tilde{x}_{1}, \ldots, \tilde{x}_{n}$ in $\widetilde{M}$ so that $Z$ has ideal $\left(x_{1}, \ldots, x_{d}\right)$ and the blow-up map $\widetilde{M} \rightarrow M$ is given by

$$
\left\{\begin{array}{c}
x_{1}=\tilde{x}_{1}, \\
x_{2}=\tilde{x}_{1} \tilde{x}_{2}, \\
\cdots \\
x_{d}=\tilde{x}_{1} \tilde{x}_{d}, \\
x_{d+1}=\tilde{x}_{d+1}, \\
\cdots \\
x_{n}=\tilde{x}_{n} .
\end{array}\right.
$$


Keeping in mind the sequences

$$
\begin{aligned}
& 0 \rightarrow \mathcal{T}^{*} M \otimes \mathcal{L} \rightarrow \mathcal{P}_{M}^{1} \mathcal{L} \rightarrow \mathcal{L} \rightarrow 0, \\
& 0 \rightarrow \mathcal{T}^{*} \widetilde{M} \otimes \mathcal{L} \rightarrow \mathcal{P} \frac{1}{M} \mathcal{L} \rightarrow \mathcal{L} \rightarrow 0,
\end{aligned}
$$

and working locally, we use $\left(s, v_{1}, \ldots, v_{n}\right)$ to denote the jet in $\mathcal{P}_{M}^{1} \mathcal{L}$ mapping to $s$ in the fibers of $\mathcal{L}$ and with differential $v_{1} d x_{1}+\cdots+v_{n} d x_{n}$. Similarly, $\left(s, \tilde{v}_{1}, \ldots, \tilde{v}_{n}\right)$ in $\mathcal{P} \frac{1}{M} \mathcal{L}$ maps to $s$ in $\mathcal{L}$ and has differential $\tilde{v}_{1} d \tilde{x}_{1}+\cdots+\tilde{v}_{n} d \tilde{x}_{n}$. The morphism

$$
\phi: \quad \mathcal{P}_{M}^{1} \mathcal{L} \rightarrow \mathcal{P}_{\widetilde{M}}^{1} \mathcal{L}
$$

defined above has the matrix

$$
\left(\begin{array}{ccccccccc}
1 & 0 & 0 & 0 & \cdots & 0 & 0 & \cdots & 0 \\
0 & 1 & \tilde{x}_{2} & \tilde{x}_{3} & \cdots & \tilde{x}_{d} & 0 & \cdots & 0 \\
0 & 0 & \tilde{x}_{1} & 0 & \cdots & 0 & 0 & \cdots & 0 \\
0 & 0 & 0 & \tilde{x}_{1} & \cdots & 0 & 0 & \cdots & 0 \\
& \vdots & & & \ddots & & & & \vdots \\
0 & 0 & 0 & 0 & \cdots & \tilde{x}_{1} & 0 & \cdots & 0 \\
0 & 0 & 0 & 0 & \cdots & 0 & 1 & \cdots & 0 \\
& \vdots & & & \vdots & & & \ddots & \vdots \\
0 & 0 & 0 & 0 & \cdots & 0 & 0 & \cdots & 1
\end{array}\right)
$$

in these coordinates. Also, the embeddings

$$
\begin{gathered}
B \ell_{\pi^{-1} Y} \widetilde{M} \subset \mathcal{P}_{M}^{1} \mathcal{L}, \\
B \ell_{Y^{\prime}}, \widetilde{M} \subset \mathcal{P} \frac{1}{\widetilde{M}} \mathcal{L}
\end{gathered}
$$

are obtained by projectivizing (and closing) the image of the sections

$$
\begin{aligned}
p & \mapsto\left(F(p),\left.\frac{\partial F}{\partial x_{1}}\right|_{p}, \ldots,\left.\frac{\partial F}{\partial x_{n}}\right|_{p}\right), \\
p & \mapsto\left(F(p),\left.\frac{\partial F}{\partial \tilde{x}_{1}}\right|_{p}, \ldots,\left.\frac{\partial F}{\partial \tilde{x}_{n}}\right|_{p}\right) .
\end{aligned}
$$

Here $F$ is the section of $\mathcal{L}$ giving the original hypersurface $X$, pulled back to $\widetilde{M}$ to give the hypersurface $X^{\prime}$. If $X$ has multiplicity $m$ along $Z, F$ will be a multiple of $\tilde{x}_{1}^{m}$ on $\widetilde{M}$.

With these notations and over points at which $F$ or some of its partials $\frac{\partial F}{\partial \tilde{x}_{i}}$ do not vanish (that is, away from $Y^{\prime}$ ), the subbundle $G_{\lambda} \subset \mathcal{P}_{M}^{1} \mathcal{L} \oplus \mathcal{P}_{\widetilde{M}}^{1} \mathcal{L}$ defined above is spanned by the $(n+1)$ rows of

$$
\left(\begin{array}{ccccccccc|ccccccccc}
\lambda & 0 & 0 & 0 & \cdots & 0 & 0 & \cdots & 0 & 1 & 0 & 0 & 0 & \cdots & 0 & 0 & \cdots & 0 \\
0 & \lambda & 0 & 0 & \cdots & 0 & 0 & \cdots & 0 & 0 & 1 & 0 & 0 & \cdots & 0 & 0 & \cdots & 0 \\
0 & 0 & \lambda & 0 & \cdots & 0 & 0 & \cdots & 0 & 0 & \tilde{x}_{2} & \tilde{x}_{1} & 0 & \cdots & 0 & 0 & \cdots & 0 \\
0 & 0 & 0 & \lambda & \cdots & 0 & 0 & \cdots & 0 & 0 & \tilde{x}_{3} & 0 & \tilde{x}_{1} & \cdots & 0 & 0 & \cdots & 0 \\
& \vdots & & & \ddots & & & & \vdots & & \vdots & & & \ddots & & & & \vdots \\
0 & 0 & 0 & 0 & \cdots & \lambda & 0 & \cdots & 0 & 0 & \tilde{x}_{d} & 0 & 0 & \cdots & \tilde{x}_{1} & 0 & \cdots & 0 \\
0 & 0 & 0 & 0 & \cdots & 0 & \lambda & \cdots & 0 & 0 & 0 & 0 & 0 & \cdots & 0 & 1 & \cdots & 0 \\
& \vdots & & & \vdots & & & \ddots & \vdots & & \vdots & & & \vdots & & & \ddots & \vdots \\
0 & 0 & 0 & 0 & \cdots & 0 & 0 & \cdots & \lambda & 0 & 0 & 0 & 0 & \cdots & 0 & 0 & \cdots & 1
\end{array}\right)
$$


together with the row vector

$$
\left(\begin{array}{lllllllllllll}
0 & 0 & 0 & 0 & \cdots & 0 & 0 & \cdots & 0 & F & \frac{\partial F}{\partial \tilde{x}_{1}} & \cdots & \frac{\partial F}{\partial \tilde{x}_{n}}
\end{array}\right)
$$

(accounting for the $0 \oplus \mathcal{O}_{\widetilde{M}}(-1)$ factor). The reason why we introduced the variety $B L$ above is to be able to extend this description to points of $Y^{\prime}$. Still working locally, give names to the sections corresponding to the various divisors on $B L$. We have:

$-E$, with local generator $\tilde{x}_{1}$ (borrowing its name from $\widetilde{M}$, from which the generator is pulled back);

- the exceptional divisors $\mathcal{Y}_{M}, \mathcal{Y}_{\widetilde{M}}$; the ideals of these are the pull-backs of $\mathcal{I}_{\pi^{-1} Y}, \mathcal{I}_{Y^{\prime}}$, and we will call local generators for these (principal) ideals $y_{M}, y_{\widetilde{M}}$ respectively;

- the exceptional divisors $\mathcal{E}_{M}, \mathcal{E}_{\widetilde{M}}$; local generators for these will be called $e_{M}$, $e_{\widetilde{M}}$ respectively.

Lemma III.2 gives the following relations among these terms:

$$
\tilde{x}_{1}=e_{M} e_{\widetilde{M}}, \quad y_{\widetilde{M}}=y_{M} e_{M}
$$

(so that also $y_{\widetilde{M}} e_{\widetilde{M}}=y_{M} \tilde{x}_{1}$ ).

Since $\mathcal{I}_{Y^{\prime}}$ pulls back to $\left(y_{\widetilde{M}}\right)$, there must be (local) $a_{0}, \ldots, a_{n}$ over $B L$ so that

$$
F=a_{0} y_{\widetilde{M}}, \quad \frac{\partial F}{\partial \tilde{x}_{1}}=a_{1} y_{\widetilde{M}}, \ldots, \frac{\partial F}{\partial \tilde{x}_{n}}=a_{n} y_{\widetilde{M}} .
$$

By the same token, there must be $b_{0}, \ldots, b_{n}$ such that

$$
F=b_{0} y_{M}, \quad \frac{\partial F}{\partial x_{1}}=b_{1} y_{M}, \ldots, \frac{\partial F}{\partial x_{n}}=b_{n} y_{M} .
$$

Further, both ideals $\left(a_{0}, \ldots, a_{n}\right)$ and $\left(b_{0}, \ldots, b_{n}\right)$ equal (1).

Now

$$
\begin{aligned}
& \frac{\partial F}{\partial \tilde{x}_{1}}=\frac{\partial F}{\partial x_{1}}+\tilde{x}_{2} \frac{\partial F}{\partial x_{2}}+\cdots+\tilde{x}_{d} \frac{\partial F}{\partial x_{d}} \\
& \frac{\partial F}{\partial \tilde{x}_{2}}=\tilde{x}_{1} \frac{\partial F}{\partial x_{2}}, \quad \ldots, \frac{\partial F}{\partial \tilde{x}_{d}}=\tilde{x}_{1} \frac{\partial F}{\partial x_{d}} \\
& \frac{\partial F}{\partial \tilde{x}_{d+1}}=\frac{\partial F}{\partial x_{d+1}}, \quad \ldots, \frac{\partial F}{\partial \tilde{x}_{n}}=\frac{\partial F}{\partial x_{n}}
\end{aligned}
$$

which translates into

$$
\begin{aligned}
a_{0} y_{\widetilde{M}}= & b_{0} y_{M}, \quad a_{1} y_{\widetilde{M}}=b_{1} y_{M}+\tilde{x}_{2} b_{2} y_{M}+\cdots+\tilde{y}_{d} b_{d} y_{M}, \\
& a_{2} y_{\widetilde{M}}=\tilde{x}_{1} b_{2} y_{M}, \quad \ldots, a_{d} y_{\widetilde{M}}=\tilde{x}_{1} b_{d} y_{M}, \\
& a_{d+1} y_{\widetilde{M}}=b_{d+1} y_{M}, \quad \ldots, a_{n} y_{\widetilde{M}}=b_{n} y_{M},
\end{aligned}
$$

and therefore

$$
\begin{gathered}
b_{0}=a_{0} e_{M}, \quad b_{1}=a_{1} e_{M}-\tilde{x}_{2} b_{2}-\cdots-\tilde{x}_{d} b_{d}, \\
a_{2}=b_{2} e_{\widetilde{M}}, \quad \ldots, a_{d}=b_{d} e_{\widetilde{M}}, \\
b_{d+1}=a_{d+1} e_{M}, \quad \ldots, b_{n}=a_{n} e_{M} .
\end{gathered}
$$

This means that we can throw away half of the $a$ 's and $b$ 's; from the data of

$$
\left\{a_{0}, a_{1}, b_{2}, \ldots, b_{d}, a_{d+1}, \ldots, a_{n}\right\}
$$


(which again locally generate (1)) we can obtain explicit coordinates in $\mathbb{P} \mathcal{P}_{M}^{1} \mathcal{L}$, $\mathbb{P} \mathcal{T} \frac{1}{M} \mathcal{L}$ for the image of a point of $B L$ :

$$
\begin{gathered}
\left(a_{0} e_{M}: a_{1} e_{M}-\tilde{x}_{2} b_{2}-\cdots-\tilde{x}_{d} b_{d}: b_{2}: \cdots: b_{d}: a_{d+1} e_{M}: \cdots: a_{n} e_{M}\right), \\
\left(a_{0}: a_{1}: b_{2} e_{\widetilde{M}}: \cdots: b_{d} e_{\widetilde{M}}: a_{d+1}: \cdots: a_{n}\right) .
\end{gathered}
$$

With this understood, the rows of the $(n+2) \times(2 n+2)$-matrix

$$
\left(\begin{array}{cccccccc|cccccccc}
\lambda & 0 & 0 & \cdots & 0 & 0 & \cdots & 0 & 1 & 0 & 0 & \cdots & 0 & 0 & \cdots & 0 \\
0 & \lambda & 0 & \cdots & 0 & 0 & \cdots & 0 & 0 & 1 & 0 & \cdots & 0 & 0 & \cdots & 0 \\
0 & 0 & \lambda & \cdots & 0 & 0 & \cdots & 0 & 0 & \tilde{x}_{2} & \tilde{x}_{1} & \cdots & 0 & 0 & \cdots & 0 \\
& \vdots & & \ddots & & & & \vdots & & \vdots & & \ddots & & & & \vdots \\
0 & 0 & 0 & \cdots & \lambda & 0 & \cdots & 0 & 0 & \tilde{x}_{d} & 0 & \cdots & \tilde{x}_{1} & 0 & \cdots & 0 \\
0 & 0 & 0 & \cdots & 0 & \lambda & \cdots & 0 & 0 & 0 & 0 & \cdots & 0 & 1 & \cdots & 0 \\
& \vdots & & \vdots & & & \ddots & \vdots & & \vdots & & \vdots & & & \ddots & \vdots \\
0 & 0 & 0 & \cdots & 0 & 0 & \cdots & \lambda & 0 & 0 & 0 & \cdots & 0 & 0 & \cdots & 1 \\
0 & 0 & 0 & \cdots & 0 & 0 & \cdots & 0 & a_{0} & a_{1} & b_{2} e_{\widetilde{M}} & \cdots & b_{d} e_{\widetilde{M}} & a_{d+1} & \cdots & a_{n}
\end{array}\right)
$$

do $\operatorname{span} G_{\lambda}$ for all $\lambda \neq 0$.

3.6. Three lemmas. The following lemmas will not be used till the final step of our proof, but this seems the best place to include them, as they use the notations we just introduced. Consider again the coordinates for a point in $B \ell_{\pi^{-1} Y} \widetilde{M} \subset \mathbb{P} \mathcal{P}_{M}^{1} \mathcal{L}$ obtained above:

$$
\left(a_{0} e_{M}: a_{1} e_{M}-\tilde{x}_{2} b_{2}-\cdots-\tilde{x}_{d} b_{d}: b_{2}: \cdots: b_{d}: a_{d+1} e_{M}: \cdots: a_{n} e_{M}\right) .
$$

The entries here are (local) components of a vector spanning $\mathcal{O}_{M}(-1)$ in $\mathcal{P}_{M}^{1} \mathcal{L}$. It is clear that the boxed entries vanish along $\mathcal{E}_{M}$ (as $e_{M}=0$ is an equation for the latter). To express this more intrinsically, consider the natural projection

$$
\rho_{Z}: \quad \mathcal{P}_{M}^{1} \mathcal{L} \oplus \mathcal{P}_{\widetilde{M}}^{1} \mathcal{L} \rightarrow \mathcal{P}_{M}^{1} \mathcal{L} \rightarrow \mathcal{P}_{Z}^{1} \mathcal{L}
$$

killing the second factor and projecting the first onto the bundle of principal parts of $\mathcal{L}$ over $Z$; then the above observation is that

Lemma III.3. $\rho_{Z}\left(\mathcal{O}_{M}(-1) \oplus 0\right)=0$ along $\mathcal{E}_{M}$.

This vanishing will be an important ingredient at the final step of the proof, in §3.8. The two lemmas that follow are also important, and they are less evident. First, observe that as $\mathcal{E}_{\widetilde{M}}$ maps injectively into $B \ell_{\pi^{-1}} \widetilde{M}$, its components either

(i) dominate $E \subset \widetilde{M}$, or

(ii) dominate components in $E \subset B \ell_{\pi^{-1}} \widetilde{M}$ which contract in $\widetilde{M}$.

Lemma III.4. $\rho_{Z}\left(\mathcal{O}_{M}(-1) \oplus 0\right)=0$ along components of $\mathcal{E}_{\widetilde{M}}$ of type (i).

Proof. To see this, it suffices to check that, at points of $\mathcal{E}_{\widetilde{M}}$ mapping to general points of $E \subset \widetilde{M}$, necessarily $e_{\widetilde{M}}$ divides $a_{0}$ and $a_{d+1}, \ldots, a_{n}$. Assume $X$ has multiplicity $m \geq 1$ along $Z$, and write (locally) $F=\tilde{x}_{1}^{m} \widetilde{F}$ (that is, let $(\widetilde{F}$ ) be the ideal of the proper transform $\widetilde{X}$ of $X$ ). Then computing partials gives the ideal of $\pi^{-1} Y$ :

$\left.{ }^{*}\right) \quad \mathcal{I}_{\pi^{-1} Y}=\tilde{x}_{1}^{m-1}\left(\tilde{x}_{1} \widetilde{F}, m \widetilde{F}+\tilde{x}_{1} \frac{\partial \widetilde{F}}{\partial \tilde{x}_{1}}, \frac{\partial \widetilde{F}}{\partial \tilde{x}_{2}}, \ldots, \frac{\partial \widetilde{F}}{\partial \tilde{x}_{d}}, \tilde{x}_{1} \frac{\partial \widetilde{F}}{\partial \tilde{x}_{d+1}}, \ldots, \tilde{x}_{1} \frac{\partial \widetilde{F}}{\partial \tilde{x}_{n}}\right)$. 
This ideal pulls back to $\left(y_{M}\right)$ in $B L$. Now $\widetilde{F} \neq 0$ at a general point of $E \subset \widetilde{M}$, and hence at a general point $p$ of the component of $\mathcal{E}_{\widetilde{M}}$ we are considering. Then at such points $(*)$ gives

$$
\mathcal{I}_{\pi^{-1} Y}=\left(\tilde{x}_{1}^{m-1}\right),
$$

that is, $y_{M}=\tilde{x}_{1}^{m-1}$ in $B L$; with the positions made in $\S 3.5$ we have

$$
a_{0} e_{M}=\tilde{x}_{1} \widetilde{F}, a_{d+1} e_{M}=\tilde{x}_{1} \frac{\partial \widetilde{F}}{\partial \tilde{x}_{d+1}}, \ldots, a_{n} e_{M}=\tilde{x}_{1} \frac{\partial \widetilde{F}}{\partial \tilde{x}_{n}},
$$

that is

$$
a_{0}=\widetilde{F} e_{\widetilde{M}}, a_{d+1}=\frac{\partial \widetilde{F}}{\partial \tilde{x}_{d+1}} e_{\widetilde{M}}, \ldots, a_{n}=\frac{\partial \widetilde{F}}{\partial \tilde{x}_{n}} e_{\widetilde{M}}
$$

$a_{0}$ and $a_{d+1}, \ldots, a_{n}$ are multiples of $e_{\widetilde{M}}$ near $p$, as we needed.

The story for components of type (ii) is a little different: the boxed entries above do not vanish identically along these components. However, let $\mathcal{E}_{\widetilde{M}}^{\prime}$ be such a component, and let $Z^{\prime}$ be the subvariety of $Z$ that $\mathcal{E}_{\widetilde{M}}^{\prime}$ dominates; also, let

$$
\mathcal{P}_{M}^{1} \mathcal{L} \oplus \mathcal{P} \frac{1}{\widetilde{M}} \mathcal{L} \stackrel{\rho_{Z^{\prime}}}{\longrightarrow} \mathcal{P}_{Z^{\prime}}^{1} \mathcal{L}
$$

denote the natural projection. Then

Lemma III.5. $\rho_{Z^{\prime}}\left(\mathcal{O}_{M}(-1) \oplus 0\right)=0$ along $\mathcal{E}_{\widetilde{M}}^{\prime}$.

Proof. Again, it suffices to show this at the general point $p$ of $\mathcal{E}_{\widetilde{M}}^{\prime}$. So let $\tilde{y}_{M}$ denote a local equation for $\mathcal{E}_{\widetilde{M}}^{\prime}$ at $p \cdot \mathcal{E}_{\widetilde{M}}^{\prime}$ dominates a component contained in $E \subset B \ell_{\pi^{-1}} \widetilde{M}$ of the exceptional divisor of the blow-up of $\widetilde{M}$ along $\pi^{-1} Y$. Thus $\mathcal{E}_{\widetilde{M}}^{\prime}$ is, aside from a multiple of $E$, the inverse image of $\pi^{-1} Y$ in $B L$ (near $p$ ), and we may assume that the ideal of the latter (again, aside from the factor $\tilde{x}_{1}^{m-1}$ ) contains $\tilde{x}_{1}$ : from $(*)$ above; we see that $\left(\tilde{y}_{M}\right)$ is the pull-back of

$$
\mathcal{J}=\left(\tilde{x}_{1}, \widetilde{F}, \frac{\partial \widetilde{F}}{\partial \tilde{x}_{2}}, \ldots, \frac{\partial \widetilde{F}}{\partial \tilde{x}_{d}}\right)
$$

near $p$, and (since $\tilde{x}_{1}^{m-1} \mathcal{J}=\mathcal{I}_{\pi^{-1} Y}$ pulls back to $\left(y_{M}\right)$ ) we have

$$
y_{M}=\tilde{x}_{1}^{m-1} \tilde{y}_{M}
$$

up to units at $p$.

Since $\tilde{x}_{1} \in \mathcal{J}$ and $\widetilde{F} \in \mathcal{J}$, we have

$$
\tilde{x}_{1}=c_{x} \tilde{y}_{M}, \quad \widetilde{F}=c_{F} \tilde{y}_{M}
$$

for some $c_{x}, c_{F}$. By the positions made in $\S 3.5$,

$$
y_{M} a_{0} e_{M}=F=\tilde{x}_{1}^{m} \widetilde{F}
$$

that is

$$
\tilde{y}_{M} a_{0} e_{M}=\tilde{x}_{1} \widetilde{F}
$$

and therefore

$$
a_{0} e_{M}=c_{x} c_{F} \tilde{y}_{M} .
$$

The left-hand side is the first boxed entry listed at the beginning of this subsection, and the right-hand side shows that this vanishes along $\mathcal{E}_{\widetilde{M}}^{\prime}$ (as $\tilde{y}_{M}=0$ is an equation 
for the latter). Behind the notational smoke, the reader should be able to see that this simply works because both $\tilde{x}_{1}$ and $\widetilde{F}$ vanish along the subscheme defined by $\mathcal{J}$ in $\widetilde{M}$; the first boxed entry is controlled by $\tilde{x}_{1} \widetilde{F}$, so it vanishes to higher order. The above computation simply formalizes this observation.

Now we want to argue similarly for the other entries. For $i=d+1, \ldots, n$ we have (from $§ 3.5$ )

$$
y_{M} a_{i} e_{M}=\frac{\partial F}{\partial \tilde{x}_{i}}=\tilde{x}_{1}^{m} \frac{\partial \widetilde{F}}{\partial \tilde{x}_{i}}
$$

and therefore

$$
\tilde{y}_{M} a_{i} e_{M}=\tilde{x}_{1} \frac{\partial \widetilde{F}}{\partial \tilde{x}_{i}}
$$

or

$$
a_{i} e_{M}=c_{x} \frac{\partial \widetilde{F}}{\partial \tilde{x}_{i}}
$$

the vanishing of $a_{i} e_{M}$ along $\mathcal{E}_{\widetilde{M}}^{\prime}$ follows for $i>d+1$ if $\frac{\partial \widetilde{F}}{\partial \tilde{x}_{i}}$ vanishes along the subset $S \subset \widetilde{M}$ dominated by $\mathcal{E}_{\widetilde{M}}^{\prime}$.

Recall we are denoting by $Z^{\prime} \subset Z$ the image of $\mathcal{E}_{\widetilde{M}}^{\prime}$ (hence of $S$ ). The image of $p$ will be a general, hence nonsingular point $q$ of $Z^{\prime}$. Choose the local parameters $\tilde{x}_{d+1}, \ldots, \tilde{x}_{n}$ on $Z$ so that $Z^{\prime}$ has equations $\tilde{x}_{d+1}=\cdots=\tilde{x}_{s}=0$ in $Z$ near $q$. Now $Z^{\prime}$ is the image of $S \subset \widetilde{F}$ : the tangent space to $\widetilde{F}$ at a general point of $S$ must dominate the tangent space to $Z^{\prime}$. This means that $\frac{\partial \widetilde{F}}{\partial \tilde{x}_{i}}=0$ along $S$ for $i=s+1, \ldots, n$, and this implies the vanishing of the corresponding entries $a_{i} e_{M}$ along $\mathcal{E}_{\widetilde{M}}^{\prime}$, as observed above. Intrinsically, this amounts to the vanishing of $\rho_{Z^{\prime}}\left(\mathcal{O}_{M}(-1) \oplus 0\right)$, and we are done.

Example. The case considered in Lemma III.5 occurs when the hypersurface is nonsingular away from $Z$, and 'cuspidal' along some subset $Z$ ' of $Z$. For an example that may help fixing ideas, consider the surface with equation

$$
x_{2}^{2}+x_{1}^{2}\left(x_{1}+x_{3}\right)=0
$$

in $\mathbb{A}^{3}$. This is singular (and equimultiple) along the line $x_{1}=x_{2}=0$; the singularity has transversal branches at all points with $x_{3} \neq 0$, but is cuspy at the origin. In the blow-up, the proper transform of the surface intersects each fiber of the exceptional divisor in two points; these collide into one point, say $r$, in the fiber over the origin. In the above terminology, $S=\{r\}$; so blowing up $\pi^{-1} Y$ in $\widetilde{M}$ amounts to blowing up $r$ in this case. Since $r \in E$, this will produce a component of $E \subset B \ell_{\pi^{-1} Y} \widetilde{M}$ contracting into $\widetilde{M}$, that is, a component of type (ii) in $\mathcal{E}_{\widetilde{M}}$. In this example $Z^{\prime}$ would be the origin.

3.7. Computing $Z_{\infty}$. In order to determine the limit of $G_{\lambda}$ as $\lambda \mapsto 0$ we consider the matrix given in $\S 3.5$ as defining a rational map

$$
B L \times \mathbb{P}^{1} \rightarrow \operatorname{Grass}_{n+2}\left(\mathcal{P}_{M}^{1} \mathcal{L} \oplus \mathcal{P}_{\widetilde{M}}^{1} \mathcal{L}\right),
$$

by sending (for $\lambda \neq 0)(p,(\lambda: 1))$ to the fiber of $G_{\lambda}$ over $p$. The plan is to resolve the indeterminacies of this map, and determine $Z_{\infty}$ as the image of specific loci via the resolved map. 
The base locus of the map is determined (using Plücker coordinates for Grass ${ }_{n+2}$ ) by the ideal of $(n+2) \times(n+2)$ minors of the matrix of row-vectors given in $\S 3.5$. In terms of the $\frac{\partial F}{\partial \tilde{x}_{i}}$ 's, this turns out to be the ideal

$$
\begin{aligned}
& \lambda^{d}\left(F, \frac{\partial F}{\partial \tilde{x}_{1}}, \ldots, \frac{\partial F}{\partial \tilde{x}_{n}}\right) \\
+ & \lambda^{d-1}\left(\tilde{x}_{1} F, \tilde{x}_{1} \frac{\partial F}{\partial \tilde{x}_{1}}, \frac{\partial F}{\partial \tilde{x}_{2}}, \ldots, \frac{\partial F}{\partial \tilde{x}_{d}}, \tilde{x}_{1} \frac{\partial F}{\partial \tilde{x}_{d+1}}, \ldots, \tilde{x}_{1} \frac{\partial F}{\partial \tilde{x}_{n}}\right) \\
+ & \ldots \\
+ & \lambda \tilde{x}^{d-2}\left(\tilde{x}_{1} F, \tilde{x}_{1} \frac{\partial F}{\partial \tilde{x}_{1}}, \frac{\partial F}{\partial \tilde{x}_{2}}, \ldots, \frac{\partial F}{\partial \tilde{x}_{d}}, \tilde{x}_{1} \frac{\partial F}{\partial \tilde{x}_{d+1}}, \ldots, \tilde{x}_{1} \frac{\partial F}{\partial \tilde{x}_{n}}\right) .
\end{aligned}
$$

Pulling back to $B L$, this is written

$$
\lambda y_{\widetilde{M}}\left(\lambda^{d-1}, \lambda^{d-2} e_{\widetilde{M}}, \lambda^{d-3} e_{M} e_{\widetilde{M}}^{2}, \ldots, e_{M}^{d-2} e_{\widetilde{M}}^{d-1}\right)
$$

and resolving the map amounts to making the ideal in ( ) principal.

By our good fortune, this is easy to accomplish: it suffices to blow-up $B L \times \mathbb{P}^{1}$ twice, first along $\mathcal{E}_{\widetilde{M}} \subset \Lambda$, where $\Lambda$ is the copy $B L \times\{(0: 1)\}$ of $B L$, and then along the proper transform of $\mathcal{E}_{M} \subset \Lambda$. In terms of ideals, first we blow-up along $\left(\lambda, e_{\widetilde{M}}\right)$; the interesting chart (we leave it to the reader to check that nothing goes wrong on the other charts) is

$$
\left\{\begin{aligned}
\lambda & =\tilde{\lambda} \tilde{e}_{\widetilde{M}}, \\
e_{\widetilde{M}} & =\tilde{e}_{\widetilde{M}},
\end{aligned}\right.
$$

so the ideal pulls back to

$$
\left(\tilde{\lambda}^{d-1} \tilde{e}_{\widetilde{M}}^{d-1}, \tilde{\lambda}^{d-2} \tilde{e}_{\widetilde{M}}^{d-1}, \tilde{\lambda}^{d-3} \tilde{e}_{\widetilde{M}}^{d-1} e_{M}, \ldots, \tilde{e}_{\widetilde{M}}^{d-1} e_{M}^{d-2}\right)=\tilde{e}_{\widetilde{M}}^{d-1}\left(\tilde{\lambda}, e_{M}\right)^{d-2}
$$

then along $\left(\tilde{\lambda}, e_{M}\right)$. It is clear that the pull-back of the ideal will then be principal, as claimed.

Now we have to do this on the matrix whose rows span the $G_{\lambda}$ 's. Again, we show what happens on one interesting chart of the result, and leave the others to the reader. We change coordinates according to

$$
\left\{\begin{array} { r l } 
{ \lambda } & { = s t , } \\
{ e _ { \widetilde { M } } , } & { = t , }
\end{array} \quad \left\{\begin{array}{rl}
s & =u v \\
e_{M} & =v
\end{array}\right.\right.
$$

that is,

$$
\left\{\begin{aligned}
\lambda & =u v t, \\
e_{M} & =v, \\
e_{\widetilde{M}} & =t, \\
\tilde{x}_{1} & =v t,
\end{aligned}\right.
$$


and the matrix becomes

$$
\left(\begin{array}{cccccccc|cccccccc}
u v t & 0 & 0 & \cdots & 0 & 0 & \cdots & 0 & 1 & 0 & 0 & \cdots & 0 & 0 & \cdots & 0 \\
0 & u v t & 0 & \cdots & 0 & 0 & \cdots & 0 & 0 & 1 & 0 & \cdots & 0 & 0 & \cdots & 0 \\
0 & 0 & u v t & \cdots & 0 & 0 & \cdots & 0 & 0 & \tilde{x}_{2} & v t & \cdots & 0 & 0 & \cdots & 0 \\
& \vdots & & \ddots & & & & \vdots & & \vdots & & \ddots & & & & \vdots \\
0 & 0 & 0 & \cdots & u v t & 0 & \cdots & 0 & 0 & \tilde{x}_{d} & 0 & \cdots & v t & 0 & \cdots & 0 \\
0 & 0 & 0 & \cdots & 0 & u v t & \cdots & 0 & 0 & 0 & 0 & \cdots & 0 & 1 & \cdots & 0 \\
& \vdots & & \vdots & & & \ddots & \vdots & & \vdots & & \vdots & & & \ddots & \vdots \\
0 & 0 & 0 & \cdots & 0 & 0 & \cdots & u v t & 0 & 0 & 0 & \cdots & 0 & 0 & \cdots & 1 \\
0 & 0 & 0 & \cdots & 0 & 0 & \cdots & 0 & a_{0} & a_{1} & b_{2} t & \cdots & b_{d} t & a_{d+1} & \cdots & a_{n}
\end{array}\right)
$$

At general points, this has the same span as

$$
\left(\begin{array}{cccccccc|cccccccc}
u v t & 0 & 0 & \cdots & 0 & 0 & \cdots & 0 & 1 & 0 & 0 & \cdots & 0 & 0 & \cdots & 0 \\
0 & u v t & 0 & \cdots & 0 & 0 & \cdots & 0 & 0 & 1 & 0 & \cdots & 0 & 0 & \cdots & 0 \\
0 & -\tilde{x}_{2} u & u & \cdots & 0 & 0 & \cdots & 0 & 0 & 0 & 1 & \cdots & 0 & 0 & \cdots & 0 \\
& \vdots & & \ddots & & & & \vdots & & \vdots & & \ddots & & & & \vdots \\
0 & -\tilde{x}_{d} u & 0 & \cdots & u & 0 & \cdots & 0 & 0 & 0 & 0 & \cdots & 1 & 0 & \cdots & 0 \\
0 & 0 & 0 & \cdots & 0 & u v t & \cdots & 0 & 0 & 0 & 0 & \cdots & 0 & 1 & \cdots & 0 \\
& \vdots & & \vdots & & & \ddots & \vdots & & \vdots & & \vdots & & & \ddots & \vdots \\
0 & 0 & 0 & \cdots & 0 & 0 & \cdots & u v t & 0 & 0 & 0 & \cdots & 0 & 0 & \cdots & 1 \\
0 & 0 & 0 & \cdots & 0 & 0 & \cdots & 0 & a_{0} & a_{1} & b_{2} t & \cdots & b_{d} t & a_{d+1} & \cdots & a_{n}
\end{array}\right)
$$

hence as

$$
\left(\begin{array}{cccccccc|cccccccc}
u v t & 0 & 0 & \cdots & 0 & 0 & \cdots & 0 & 1 & 0 & 0 & \cdots & 0 & 0 & \cdots & 0 \\
0 & u v t & 0 & \cdots & 0 & 0 & \cdots & 0 & 0 & 1 & 0 & \cdots & 0 & 0 & \cdots & 0 \\
0 & -\tilde{x}_{2} u & u & \cdots & 0 & 0 & \cdots & 0 & 0 & 0 & 1 & \cdots & 0 & 0 & \cdots & 0 \\
& \vdots & & \ddots & & & & \vdots & & \vdots & & \ddots & & & & \vdots \\
0 & -\tilde{x}_{d} u & 0 & \cdots & u & 0 & \cdots & 0 & 0 & 0 & 0 & \cdots & 1 & 0 & \cdots & 0 \\
0 & 0 & 0 & \cdots & 0 & u v t & \cdots & 0 & 0 & 0 & 0 & \cdots & 0 & 1 & \cdots & 0 \\
& \vdots & & \vdots & & & \ddots & \vdots & & \vdots & & \vdots & & & \ddots & \vdots \\
0 & 0 & 0 & \cdots & 0 & 0 & \cdots & u v t & 0 & 0 & 0 & \cdots & 0 & 0 & \cdots & 1 \\
a_{0} v & a_{1} v-\sum_{2}^{d} \tilde{x}_{i} b_{i} & b_{2} & \cdots & b_{d} & a_{d+1} v & \cdots & a_{n} v & 0 & 0 & 0 & \cdots & 0 & 0 & \cdots & 0
\end{array}\right)
$$

This matrix has maximal rank everywhere, as it ought to (this is because

$$
\left(a_{0} v, a_{1} v-\sum_{2}^{d} \tilde{x}_{i} b_{i}, b_{2}, \cdots, b_{d}, a_{d+1} v, \cdots, a_{n} v\right)=(1)
$$

cf. $\S 3.5)$; so the map corresponding to $\operatorname{Grass}_{n+2}$ is indeed defined everywhere. The $\lim _{\lambda \mapsto 0} G_{\lambda}$ is therefore determined by the image of $\lambda=0$; since $\lambda=u v t$, this breaks up the limit into three pieces: 
- over $u=0$, the component dominating $B L \times\{(0: 1)\}$; this gives

$$
\left(\begin{array}{cccccccc|cccccccc}
0 & 0 & 0 & \cdots & 0 & 0 & \cdots & 0 & 1 & 0 & 0 & \cdots & 0 & 0 & \cdots & 0 \\
0 & 0 & 0 & \cdots & 0 & 0 & \cdots & 0 & 0 & 1 & 0 & \cdots & 0 & 0 & \cdots & 0 \\
0 & 0 & 0 & \cdots & 0 & 0 & \cdots & 0 & 0 & 0 & 1 & \cdots & 0 & 0 & \cdots & 0 \\
& \vdots & & \ddots & & & & \vdots & & \vdots & & \ddots & & & & \vdots \\
0 & 0 & 0 & \cdots & 0 & 0 & \cdots & 0 & 0 & 0 & 0 & \cdots & 1 & 0 & \cdots & 0 \\
0 & 0 & 0 & \cdots & 0 & 0 & \cdots & 0 & 0 & 0 & 0 & \cdots & 0 & 1 & \cdots & 0 \\
& \vdots & & \vdots & & & \ddots & \vdots & & \vdots & & \vdots & & & \ddots & \vdots \\
0 & 0 & 0 & \cdots & 0 & 0 & \cdots & 0 & 0 & 0 & 0 & \cdots & 0 & 0 & \cdots & 1 \\
a_{0} v & a_{1} v-\sum_{2}^{d} \tilde{x}_{i} b_{i} & b_{2} & \cdots & b_{d} & a_{d+1} v & \cdots & a_{n} v & 0 & 0 & 0 & \cdots & 0 & 0 & \cdots & 0
\end{array}\right)
$$

which projectivizes to $\left[\mathbb{P}\left(\mathcal{O}_{M}(-1) \oplus \mathcal{P} \frac{1}{\widetilde{M}} \mathcal{L}\right)\right]=\left[G_{M}\right]$ (as we promised in $\S 3.4, G_{M}$ had to appear as one component in the limit);

- over $v=0$, which dominates $\mathcal{E}_{M} \times\{(0: 1)\}$ :

$$
\left(\begin{array}{cccccccc|cccccccc}
0 & 0 & 0 & \cdots & 0 & 0 & \cdots & 0 & 1 & 0 & 0 & \cdots & 0 & 0 & \cdots & 0 \\
0 & 0 & 0 & \cdots & 0 & 0 & \cdots & 0 & 0 & 1 & 0 & \cdots & 0 & 0 & \cdots & 0 \\
0 & -\tilde{x}_{2} u & u & \cdots & 0 & 0 & \cdots & 0 & 0 & 0 & 1 & \cdots & 0 & 0 & \cdots & 0 \\
& \vdots & & \ddots & & & & \vdots & & \vdots & & \ddots & & & & \vdots \\
0 & -\tilde{x}_{d} u & 0 & \cdots & u & 0 & \cdots & 0 & 0 & 0 & 0 & \cdots & 1 & 0 & \cdots & 0 \\
0 & 0 & 0 & \cdots & 0 & 0 & \cdots & 0 & 0 & 0 & 0 & \cdots & 0 & 1 & \cdots & 0 \\
& \vdots & & \vdots & & & \ddots & \vdots & & \vdots & & \vdots & & & \ddots & \vdots \\
0 & 0 & 0 & \cdots & 0 & 0 & \cdots & 0 & 0 & 0 & 0 & \cdots & 0 & 0 & \cdots & 1 \\
0 & -\sum_{2}^{d} \tilde{x}_{i} b_{i} & b_{2} & \cdots & b_{d} & 0 & \cdots & 0 & 0 & 0 & 0 & \cdots & 0 & 0 & \cdots & 0
\end{array}\right)
$$

-and over $t=0$, which dominates $\mathcal{E}_{\widetilde{M}} \times\{(0: 1)\}$ :

$$
\left(\begin{array}{cccccccc|cccccccc}
0 & 0 & 0 & \cdots & 0 & 0 & \cdots & 0 & 1 & 0 & 0 & \cdots & 0 & 0 & \cdots & 0 \\
0 & 0 & 0 & \cdots & 0 & 0 & \cdots & 0 & 0 & 1 & 0 & \cdots & 0 & 0 & \cdots & 0 \\
0 & -\tilde{x}_{2} u & u & \cdots & 0 & 0 & \cdots & 0 & 0 & 0 & 1 & \cdots & 0 & 0 & \cdots & 0 \\
& \vdots & & \ddots & & & & \vdots & & \vdots & & \ddots & & & & \vdots \\
0 & -\tilde{x}_{d} u & 0 & \cdots & u & 0 & \cdots & 0 & 0 & 0 & 0 & \cdots & 1 & 0 & \cdots & 0 \\
0 & 0 & 0 & \cdots & 0 & 0 & \cdots & 0 & 0 & 0 & 0 & \cdots & 0 & 1 & \cdots & 0 \\
& \vdots & & \vdots & & & \ddots & \vdots & & \vdots & & \vdots & & & \ddots & \vdots \\
0 & 0 & 0 & \cdots & 0 & 0 & \cdots & 0 & 0 & 0 & 0 & \cdots & 0 & 0 & \cdots & 1 \\
a_{0} v & a_{1} v-\sum_{2}^{d} \tilde{x}_{i} b_{i} & b_{2} & \cdots & b_{d} & a_{d+1} v & \cdots & a_{n} v & 0 & 0 & 0 & \cdots & 0 & 0 & \cdots & 0
\end{array}\right)
$$

These last two loci make up $Z_{\infty}$ (by definition of the latter as residual of $G_{M}$ in the limit). We have to study these loci, aiming toward computing the class of the statement of Claim III.3.

3.8. End of the proof of (3). Summarizing, we have determined $Z_{\infty}$ as the image of two loci defined over a double blow-up of $B L \times \mathbb{P}^{1}$. They both sit in (the pull-back of) $\mathbb{P}\left(\mathcal{P}_{M}^{1} \mathcal{L} \oplus \mathcal{P} \widetilde{M}_{\widetilde{L}}^{1} \mathcal{L}\right)$, and they dominate respectively the second and first exceptional divisors (equations $v=0, t=0$ ), which we will call $\mathcal{D}_{M}, \mathcal{D}_{\widetilde{M}}$; these in turn dominate resp. $\mathcal{E}_{M}, \mathcal{E}_{\widetilde{M}}$. The loci can be written as projectivizations $F_{M}=\mathbb{P} \mathcal{F}_{M}, F_{\widetilde{M}}=\mathbb{P} \mathcal{F}_{\widetilde{M}}$ of rank- $(n+2)$ subbundles $\mathcal{F}_{M}, \mathcal{F}_{\widetilde{M}}$ of $\mathcal{P}_{M}^{1} \mathcal{L} \oplus \mathcal{P}_{\widetilde{M}}^{1} \mathcal{L}$ over 
resp. $\mathcal{D}_{M}, \mathcal{D}_{\widetilde{M}}$, determined by the last two matrices written above. The following is our final reformulation of (3):

Claim III.4. In order to prove (3), it suffices to show that

$$
c\left(\frac{\mathcal{P}_{M}^{1} \mathcal{L} \oplus \mathcal{P}_{\widetilde{M}}^{1} \mathcal{L}}{\mathcal{O}(-1)}\right) \cap\left[F_{M}\right], \quad c\left(\frac{\mathcal{P}_{M}^{1} \mathcal{L} \oplus \mathcal{P}_{\widetilde{M}}^{1} \mathcal{L}}{\mathcal{O}(-1)}\right) \cap\left[F_{\widetilde{M}}\right]
$$

vanish after push-forward to $M$.

Proof. Via the map of bundles

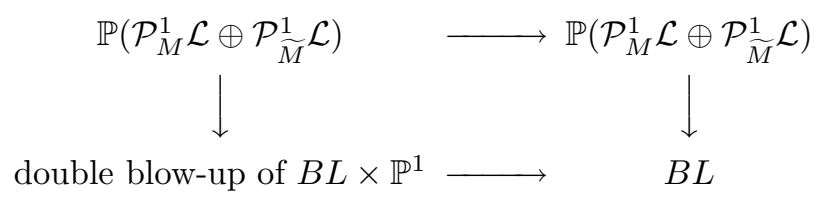

the cycle $\left[F_{M}\right]+\left[F_{\widetilde{M}}\right]$ (which lives in the top-left spot) pushes forward to $\left[Z_{\infty}\right]$ (in the top-right spot). So the claim follows directly from Claim III.3.

Finally, we are ready to complete the proof of (3), and therefore of the main theorem:

Proof of (3). Observe that

$$
c\left(\frac{\mathcal{P}_{M}^{1} \mathcal{L} \oplus \mathcal{P}_{\widetilde{M}}^{1} \mathcal{L}}{\mathcal{O}(-1)}\right) \cap\left[F_{M}\right]=c\left(\frac{\mathcal{P}_{M}^{1} \mathcal{L} \oplus \mathcal{P}_{\widetilde{M}}^{1} \mathcal{L}}{\mathcal{F}_{M}}\right) c\left(\frac{\mathcal{F}_{M}}{\mathcal{O}(-1)}\right) \cap\left[F_{M}\right]
$$

pushes forward to

$$
c\left(\frac{\mathcal{P}_{M}^{1} \mathcal{L} \oplus \mathcal{P}_{\widetilde{M}}^{1} \mathcal{L}}{\mathcal{F}_{M}}\right) \cap\left[\mathcal{D}_{M}\right]
$$

on the double blow-up of $B L \times \mathbb{P}^{1}$; indeed, $\mathcal{O}(-1)$ restricts to the universal line bundle in $\mathcal{F}_{M}$, so $c(\mathcal{O}(-1))^{-1} \cap\left[F_{M}\right]$ pushes forward to $c\left(\mathcal{F}_{M}\right)^{-1} \cap\left[\mathcal{D}_{M}\right]$. Similarly

$$
c\left(\frac{\mathcal{P}_{M}^{1} \mathcal{L} \oplus \mathcal{P}_{\widetilde{M}}^{1} \mathcal{L}}{\mathcal{O}(-1)}\right) \cap\left[F_{\widetilde{M}}\right]
$$

pushes forward to

$$
c\left(\frac{\mathcal{P}_{M}^{1} \mathcal{L} \oplus \mathcal{P}_{\widetilde{M}}^{1} \mathcal{L}}{\mathcal{F}_{\widetilde{M}}}\right) \cap\left[\mathcal{D}_{\widetilde{M}}\right]
$$

The reason why these classes $(\dagger),(\dagger \dagger)$ vanish when pushed forward to $M$ lies in the three lemmas in §3.6. Arguing explicitly for $(\dagger)$, the key observation is that $\mathcal{F}_{M}$ is contained in the kernel of the natural morphism $\mathcal{P}_{M}^{1} \mathcal{L} \oplus \mathcal{P}_{\widetilde{M}}^{1} \mathcal{L} \stackrel{\rho_{Z}}{\longrightarrow} \mathcal{P}_{Z}^{1} \mathcal{L}$ : this can be checked locally, and it is immediate from the matrix description given above, since in the chosen coordinates $\rho_{Z}$ acts by

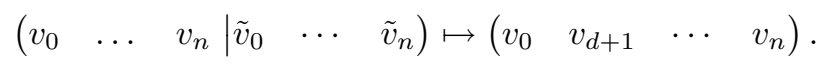

Lemma III.3 in $\S 3.6$ amounts to observing this vanishing for the last row of the matrix, as $\mathcal{D}_{M}$ dominates $\mathcal{E}_{M}$; the vanishing for the rest of the matrix is clear for $(\dagger)$ as well as for $(\dagger \dagger)$. 
Therefore we have an onto morphism

$$
\frac{\mathcal{P}_{M}^{1} \mathcal{L} \oplus \mathcal{P}_{\widetilde{M}}^{1} \mathcal{L}}{\mathcal{F}_{M}} \rightarrow \mathcal{P}_{Z}^{1} \mathcal{L} \rightarrow 0
$$

if $\mathcal{K}$ denotes the kernel of this morphism, and $\Pi$ denotes the projection to $M$, we get

$$
\Pi_{*}\left(c\left(\frac{\mathcal{P}_{M}^{1} \mathcal{L} \oplus \mathcal{P}_{\widetilde{M}}^{1} \mathcal{L}}{\mathcal{F}_{M}}\right) \cap\left[\mathcal{D}_{M}\right]\right)=c\left(\mathcal{P}_{Z}^{1} \mathcal{L}\right) \Pi_{*}\left(c(\mathcal{K}) \cap\left[\mathcal{D}_{M}\right]\right) .
$$

Now $\mathcal{D}_{M}$ has dimension $n\left(\mathcal{D}_{M}\right.$ is a divisor in a blow-up of $B L \times \mathbb{P}^{1}$, and $B L$ is birational to $M)$ while $\mathcal{K}$ has rank $(2 n+2)-(n+2)-(n-d+1)=d-1$, so that $c(\mathcal{K}) \cap\left[\mathcal{D}_{M}\right]$ has no terms in dimension $\leq \operatorname{dim} Z$, while $\mathcal{D}_{M}$ dominates $Z$ via $\Pi$ : $\mathcal{D}_{M}$ dominates $\mathcal{E}_{M}$, then $E$, then $Z$. This forces the last $\Pi_{*}$ to vanish, as needed.

Concerning (††), Lemma III.4 in $\S 3.6$ shows that $\mathcal{F}_{\widetilde{M}}$ is in the kernel of $\rho_{Z}$ along components dominating components 'of type (i)' of $\mathcal{E}_{\widetilde{M}}$, and the vanishing follows by the same argument as for $(\dagger)$.

The situation is slightly more complicated over components 'of type (ii)'. By Lemma III.5 in $\S 3.6$ we know that, along such a component $\mathcal{D}_{\widetilde{M}}^{\prime}, \mathcal{F}_{\widetilde{M}}$ is in the kernel of the epimorphism $\rho_{Z^{\prime}}: \mathcal{P}_{M}^{1} \mathcal{L} \oplus \mathcal{P}_{\widetilde{M}}^{1} \mathcal{L} \rightarrow \mathcal{P}_{Z^{\prime}}^{1} \mathcal{L}$ for the subvariety $Z^{\prime}$ of $Z$ dominated by the component. Now pull back the situation through the fiber square

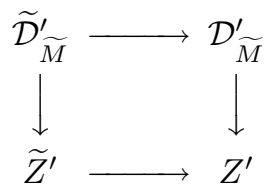

where the bottom row is the Nash-blow-up of $Z^{\prime}$ : over $\widetilde{Z}^{\prime}$, the pull-back of $\mathcal{P}_{Z^{\prime}}^{1} \mathcal{L}$ surjects onto a locally free sheaf of $\operatorname{rank} 1+\operatorname{dim} Z^{\prime}$; the above argument then tells us that

$$
\left(c\left(\frac{\mathcal{P}_{M}^{1} \mathcal{L} \oplus \mathcal{P}_{\widetilde{M}}^{1} \mathcal{L}}{\mathcal{F}_{\widetilde{M}}}\right) \cap\left[\widetilde{\mathcal{D}}_{\widetilde{M}}^{\prime}\right]\right)
$$

vanishes after push-forward to $\widetilde{Z}^{\prime}$, and this implies the vanishing of

$$
c\left(\frac{\mathcal{P}_{M}^{1} \mathcal{L} \oplus \mathcal{P}_{\widetilde{M}}^{1} \mathcal{L}}{\mathcal{F}_{\widetilde{M}}}\right) \cap\left[\mathcal{D}_{\widetilde{M}}^{\prime}\right]
$$

after push-forward to $Z^{\prime} \subset M$, as needed. This concludes the proof of (3), in the equivalent formulation stated in Claim III.4.

\section{REMARKS AND EXAMPLes}

4.1. $\mu$-class and Parusiński's Milnor number. Assuming $X$ is complete and taking degrees in Theorem I.5 gives

$$
\int c_{\mathrm{SM}}(X)=\int \frac{c(T M)}{c(\mathcal{L})} \cap[X]+\int c(\mathcal{L})^{\operatorname{dim} X} \cap\left(\mu_{\mathcal{L}}(Y)^{\vee} \otimes_{M} \mathcal{L}\right) .
$$

Now observe that the $\int$ picks up the term of degree $\operatorname{dim} M=\operatorname{dim} X+1$ in the last term. Thinking of $c(\mathcal{L})^{\operatorname{dim} X}$ as $c\left(\mathcal{L}^{\oplus \operatorname{dim} X}\right.$ ) and using (an immediate generalization 
of) $[\mathrm{A}-\mathrm{F}]$, we get

$$
\begin{aligned}
\int c(\mathcal{L})^{\operatorname{dim} X} \cap\left(\mu_{\mathcal{L}}(Y)^{\vee} \otimes \mathcal{L}\right) & =\int c\left(\mathcal{L}^{\oplus \operatorname{dim} X} \otimes \mathcal{L}^{\vee}\right) \cap\left(\mu_{\mathcal{L}}(Y)^{\vee} \otimes \mathcal{L} \otimes \mathcal{L}^{\vee}\right) \\
& =\int \mu_{\mathcal{L}}(Y)^{\vee} .
\end{aligned}
$$

Recalling that the degree of $c_{\mathrm{SM}}(X)$ equals the Euler characteristic of $X$, this proves

Proposition IV.1.

$$
\int \mu_{\mathcal{L}}(Y)=(-1)^{\operatorname{dim} M}\left(\chi(X)-\int \frac{c(T M)}{c(\mathcal{L})} \cap[X]\right) .
$$

Over $\mathbb{C}$, the right-hand-side in this formula equals Parusiński's generalization of Milnor's number ([Parusiński1]); so this gives an alternative proof of Proposition 2.1 in [Aluffi1], and extends to arbitrary fields of characteristic 0 the interpretation of $\int \mu_{\mathcal{L}}(Y)$ as a measure of the difference in the Euler characteristics of special vs. general sections of a line bundle (if $\mathcal{L}$ has enough sections, the last term in $\left(^{*}\right.$ ) gives $\chi\left(X_{g}\right)$ for a general section $X_{g}$ of $\left.\mathcal{L}\right)$.

Conversely, at least if $\mathcal{L}$ is ample enough, the formula in Proposition IV.1 suffices to prove Theorem I 'numerically', that is, up to taking degrees with respect to $\mathcal{L}$. This is worked out in [Aluffi2].

For isolated singularities on strong local complete intersections, a statement analogous to Theorem I.5 has been proved by T. Suwa [Suwa].

4.2. Blowing up $\mu$-classes. The blow-up formula proved in $\S 3$ translates nicely in terms of $\mu$-classes.

Notations as in (3) from $\S 2: Z \subset X \subset M$ is a nonsingular subvariety of codimension $d$ in $M(\operatorname{dim} M=n), \widetilde{M}=B \ell_{Z} M \rightarrow M$ denotes the blow-up of $M$ along $Z$, and $X^{\prime}$ denotes the (scheme-theoretic) inverse image of $X$ in $\widetilde{M}$. Also, $Y, Y^{\prime}$ are the singular schemes of $X, X^{\prime}$ respectively. If $\mathcal{L}$ denotes the line bundle of $X$, note that its pull-back is the line-bundle of $X^{\prime}$. Then the equality (3) we proved in $\S 3$,

$$
\pi_{*}\left(c_{*}\left(X^{\prime}\right)\right)=c_{*}(X)+(d-1) c_{*}(Z)
$$

(with $c_{*}$ as in $\S 1$ ), becomes, in terms of $\mu$-classes,

Proposition IV.2. With notations as above,

$$
\pi_{*} \mu_{\mathcal{L}}\left(Y^{\prime}\right)=\mu_{\mathcal{L}}(Y)+(-1)^{d}(d-1) \mu_{\mathcal{L}}(Z)
$$

in $A_{*} X$.

Note: it is reasonable to expect that this equality holds in $A_{*}(Y \cup Z)$.

Proof. Since $\mathcal{O}\left(X^{\prime}\right)$ is the pull-back of $\mathcal{O}(X)$,

$$
\begin{aligned}
\pi_{*}(c(T \widetilde{M}) & \left.\cap s\left(X^{\prime}, \widetilde{M}\right)\right)-c(T M) \cap s(X, M) \\
& =\pi_{*}\left(c(T \widetilde{M}) \cap \frac{\pi^{*}[X]}{1+\pi^{*} X}\right)-c(T M) \cap \frac{[X]}{1+X} \\
& =\left(\pi_{*}(c(T \widetilde{M}) \cap[\widetilde{M}])-c(T M) \cap[M]\right) \cdot \frac{[X]}{1+X} .
\end{aligned}
$$

Now we already observed in $\S 3.1$ that

$$
\pi_{*}(c(T \widetilde{M}) \cap[\widetilde{M}])-c(T M) \cap[M]=(d-1) c(T Z) \cap[Z] ;
$$


therefore, using the expression for $c_{*}$ in Theorem I.5, (3) is equivalent to

$$
\begin{aligned}
\pi_{*}\left(c(\mathcal{L})^{n-1} \cap\left(\mu_{\mathcal{L}}\left(Y^{\prime}\right)^{\vee} \otimes \mathcal{L}\right)\right)-c(\mathcal{L})^{n-1} \cap\left(\mu_{\mathcal{L}}(Y)^{\vee} \otimes \mathcal{L}\right) \\
\quad=(d-1) c(T Z) \cap[Z]-(d-1) c(T Z) \cap[Z] \cdot \frac{[X]}{1+X},
\end{aligned}
$$

that is, to

$$
\pi_{*}\left(c(\mathcal{L})^{n-1} \cap\left(\mu_{\mathcal{L}}\left(Y^{\prime}\right)^{\vee} \otimes \mathcal{L}\right)\right)-c(\mathcal{L})^{n-1} \cap\left(\mu_{\mathcal{L}}(Y)^{\vee} \otimes \mathcal{L}\right)=(d-1) \frac{c(T Z)}{c(\mathcal{L})} \cap[Z]
$$

Now we apply easy manipulations (see [Aluffi2], §2):

- cap by $c(\mathcal{L})^{-(n-1)}$ :

$$
\begin{gathered}
\pi_{*} \mu_{\mathcal{L}}\left(Y^{\prime}\right)^{\vee} \otimes \mathcal{L}-\mu_{\mathcal{L}}(Y)^{\vee} \otimes \mathcal{L}=(d-1) \frac{c(T Z)}{c(\mathcal{L})^{n}} \cap[Z] ; \\
-\otimes_{M} \mathcal{L}^{\vee}: \\
\pi_{*} \mu_{\mathcal{L}}\left(Y^{\prime}\right)^{\vee}-\mu_{\mathcal{L}}(Y)^{\vee}=(d-1) c\left(\mathcal{L}^{\vee}\right)^{d} \frac{c\left(T Z \otimes \mathcal{L}^{\vee}\right)}{c\left(\mathcal{L} \otimes \mathcal{L}^{\vee}\right)^{n}} \cap \frac{[Z]}{c\left(\mathcal{L}^{\vee}\right)^{d}} ;
\end{gathered}
$$

— clean up, dualize, apply Corollary 1.8 from [Aluffi1]:

$$
\pi_{*} \mu_{\mathcal{L}}\left(Y^{\prime}\right)-\mu_{\mathcal{L}}(Y)=(-1)^{d}(d-1) c\left(T^{*} Z \otimes \mathcal{L}\right) \cap[Z]=(-1)^{d}(d-1) \mu_{\mathcal{L}}(Z),
$$

as needed.

The relation of $\mu$-classes stated above is of some independent interest. It is related to a result in [Parusiński1] (Lemma 2.2), which can be stated as the fact that (if $X$ is complete) the zero-dimensional terms of the two sides have the same degree.

4.3. Contact of two hypersurfaces. Our proof of the main theorem used very little of the good functoriality properties of $c_{\mathrm{SM}}(X)$ : we proved just enough of them for $c_{*}(X)$ to force this to equal $c_{\mathrm{SM}}(X)$. After the fact, however, $c_{*}(X)$ inherits the full set from $c_{\mathrm{SM}}(X)$, and this reflects into facts about Segre classes of singular schemes of hypersurfaces which we are not able to prove otherwise, or which would require substantially more work by more conventional techniques. While we plan to explore this elsewhere, we give a few such examples in this subsection.

Suppose $M_{1}, M_{2}$ are distinct nonsingular hypersurfaces of a nonsingular ambient variety $M$. Then $X=M_{1} \cap M_{2}$ is a hypersurface of both $M_{1}$ and $M_{2}$, with normal bundle $\mathcal{L}_{2}=\left.\mathcal{O}\left(M_{2}\right)\right|_{X}$ in $M_{1}$ and $\mathcal{L}_{1}=\left.\mathcal{O}\left(M_{1}\right)\right|_{X}$ in $M_{2}$. The singular scheme $Y$ of $X$ is supported on the locus where $M_{1}$ and $M_{2}$ are tangent: we call $Y$ the contact scheme of $M_{1}$ and $M_{2}$ in this case. What can be said in general about $Y$ ?

Proposition IV.3. Under the above hypotheses (and with the notation introduced in $§ 1.4)$,

$$
s\left(Y, M_{1}\right) \otimes_{M_{1}} \mathcal{L}_{1}=s\left(Y, M_{2}\right) \otimes_{M_{2}} \mathcal{L}_{2}
$$

Again, while it is reasonable to expect that this equality holds in $A_{*} Y$, our argument only proves it in $A_{*} X$.

Proof. By Theorem I.4, we can compute $c_{\mathrm{SM}}(X)$ by viewing it as a hypersurface of $M_{1}$ :

$$
c_{\mathrm{SM}}(X)=c\left(T M_{1}\right) \cap\left(s\left(X, M_{1}\right)+c\left(\mathcal{L}_{2}\right)^{-1} \cap\left(s\left(Y, M_{1}\right)^{\vee} \otimes_{M_{1}} \mathcal{L}_{2}\right)\right),
$$


or as a hypersurface of $M_{2}$ :

$$
c_{\mathrm{SM}}(X)=c\left(T M_{2}\right) \cap\left(s\left(X, M_{2}\right)+c\left(\mathcal{L}_{1}\right)^{-1} \cap\left(s\left(Y, M_{2}\right)^{\vee} \otimes_{M_{2}} \mathcal{L}_{1}\right)\right) .
$$

It follows that the right-hand sides of these expressions are equal. The first summand in both is $c_{F}(X)$, so we get

$$
\frac{c\left(T M_{1}\right)}{c\left(\mathcal{L}_{2}\right)} \cap\left(s\left(Y, M_{1}\right)^{\vee} \otimes_{M_{1}} \mathcal{L}_{2}\right)=\frac{c\left(T M_{2}\right)}{c\left(\mathcal{L}_{1}\right)} \cap\left(s\left(Y, M_{2}\right)^{\vee} \otimes_{M_{2}} \mathcal{L}_{1}\right) .
$$

Capping with the inverse Chern class of the virtual tangent bundle of $X$ and dualizing yields

$$
s\left(Y, M_{1}\right) \otimes_{M_{1}} \mathcal{L}_{2}^{\vee}=s\left(Y, M_{2}\right) \otimes_{M_{2}} \mathcal{L}_{1}^{\vee} .
$$

Tensoring both sides by $\mathcal{L}_{1} \otimes \mathcal{L}_{2}$ gives the statement of the proposition.

We do not see any simple way to derive the result in Proposition IV.3 more directly. The result prompts us to define a class

$$
\mathcal{S}(Y, M)=s\left(Y, M_{1}\right) \otimes_{M_{1}} \mathcal{L}_{1},
$$

since we just showed that this is in a sense intrinsic to the contact scheme and to the ambient variety. It would be interesting to study properties of this class.

Next, observe that $M_{1} \cup M_{2}$ is a hypersurface of $M$, with line bundle $\mathcal{O}\left(M_{1}\right) \otimes$ $\mathcal{O}\left(M_{2}\right)$. The singular scheme $\bar{X}$ of $M_{1} \cup M_{2}$ is supported on $X=M_{1} \cap M_{2}$, but 'thicker' than $X$ along $Y$. Now

$$
c_{\mathrm{SM}}\left(M_{1} \cup M_{2}\right)=c_{\mathrm{SM}}\left(M_{1}\right)+c_{\mathrm{SM}}\left(M_{2}\right)-c_{\mathrm{SM}}\left(M_{1} \cap M_{2}\right)
$$

(cf. the beginning of $\S 2$ ). If the hypersurfaces involved are all divisors with normal crossings, we proved this relation 'by hand' in $\S 2$ for the class $c_{*}$ defined in $\S 1$. As we have now proved that $c_{*}=c_{\mathrm{SM}}$, we know that this equality must hold regardless of how the hypersurfaces meet. Using for example the expression for $c_{*}$ given in Theorem I.4, this gives a nontrivial relation among $s(\bar{X}, M)$ (on the left-hand side) and $s\left(Y, M_{i}\right)$ (on the right-hand side). Unraveling notations, the reader will check that this gives (in $A_{*}\left(M_{1} \cup M_{2}\right)$ )

Proposition IV.4. $s(\bar{X}, M)=s(X, M)+c\left(N_{X} M\right)^{-1} \cap \mathcal{S}(Y, M)$.

This is a sort of 'residual intersection formula' (thinking of $Y$ as the residual of $X$ in $\bar{X}$ ), and as such it could probably be proved by judicious use of Proposition 9.2 in [Fulton], perhaps after blowing up $M$ along $X$. If $\bar{X} \subset M_{1}$, then standard residual intersection formulas can be applied to $X \subset \bar{X} \subset M_{1}$ (as $X$ is a divisor of $M_{1}$ ), and do yield the formula stated in the proposition. However, in general $\bar{X}$ is not contained in either $M_{1}$ or $M_{2}$.

The above argument will work even if one of the hypersurfaces, say $M_{2}$, is singular. In such a case $\bar{X}$ will be supported on $X=M_{1} \cap M_{2}$ and on $W=$ the singular scheme of $M_{2}$. In terms of $\left.c_{*},{ }^{* *}\right)$ then says (all $\otimes$ in $M$ unless otherwise denoted)

$$
\begin{aligned}
& c(T M) \cap(\left.\frac{\left[M_{1}\right]+\left[M_{2}\right]}{c\left(\mathcal{L}_{1} \otimes \mathcal{L}_{2}\right)}+\frac{1}{c\left(\mathcal{L}_{1} \otimes \mathcal{L}_{2}\right)} \cap\left(s(\bar{X}, M)^{\vee} \otimes \mathcal{L}_{1} \otimes \mathcal{L}_{2}\right)\right) \\
&=c(T M) \cap\left(\frac{\left[M_{1}\right]}{c\left(\mathcal{L}_{1}\right)}+\frac{\left[M_{2}\right]}{c\left(\mathcal{L}_{2}\right)}+\frac{1}{c\left(\mathcal{L}_{2}\right)} \cap\left(s(W, M)^{\vee} \otimes \mathcal{L}_{2}\right)\right) \\
& \quad-c\left(T M_{1}\right) \cap\left(\frac{\left[M_{1}\right] \cdot\left[M_{2}\right]}{c\left(\mathcal{L}_{2}\right)}-\frac{1}{c\left(\mathcal{L}_{2}\right)}\left(s\left(Y, M_{1}\right)^{\vee} \otimes_{M_{1}} \mathcal{L}_{2}\right)\right)
\end{aligned}
$$


(note: the last $\vee$ is also taken in $M$, hence the change of sign in the last ()). The reader should have no difficulties (using [Aluffi2]) simplifying this expression to

Proposition IV.5. With the above notations,

$$
s(\bar{X}, M)-s(X, M)=\frac{1}{c\left(\mathcal{L}_{1}\right)} \cap\left(s(W, M) \otimes_{M} \mathcal{L}_{1}\right)+\frac{1}{c\left(\mathcal{L}_{2}\right)} \cap\left(s\left(Y, M_{1}\right) \otimes_{M} \mathcal{L}_{1}\right)
$$

(again, in $A_{*}\left(M_{1} \cup M_{2}\right)$ ).

The residual intersection problem is in this case complicated enough that we were not able to prove this relation otherwise.

4.4. A geometric application. If the singular scheme $Y$ of a hypersurface $X$ is nonsingular, then the class $c_{*}(X)$ of $\S 1$ (and hence $c_{\mathrm{SM}}(X)$ ) has a particularly simple form:

Proposition IV.6. Let $X$ be a hypersurface in a nonsingular variety $M$, let $\mathcal{L}=$ $\mathcal{O}(X)$ and assume that the singular scheme $Y$ of $X$ is nonsingular. Then

$$
c_{\mathrm{SM}}(X)=c_{F}(X)+(-1)^{\operatorname{codim}_{M} Y} \frac{c(T Y)}{c(\mathcal{L})} \cap[Y] .
$$

Proof. By [Aluffi1], Corollary 1.8, if $Y$ is nonsingular then

$$
\mu_{\mathcal{L}}(Y)=c\left(T^{*} Y \otimes \mathcal{L}\right) \cap[Y]
$$

hence by Theorem I.5

$$
c_{\mathrm{SM}}(X)=c_{F}(X)+c(\mathcal{L})^{\operatorname{dim} X} \cap\left(\left(c\left(T^{*} Y \otimes \mathcal{L}\right) \cap[Y]\right)^{\vee} \otimes_{M} \mathcal{L}\right) .
$$

Using [Aluffi2], §2, we get

$$
\begin{aligned}
c(\mathcal{L})^{\operatorname{dim} X} & \cap\left(\left(c\left(T^{*} Y \otimes \mathcal{L}\right) \cap[Y]\right)^{\vee} \otimes_{M} \mathcal{L}\right) \\
= & (-1)^{\operatorname{codim}_{M} Y} c(\mathcal{L})^{\operatorname{dim} X}\left(\left(c\left(T Y \otimes \mathcal{L}^{\vee}\right) \cap[Y]\right) \otimes_{M} \mathcal{L}\right) \\
& =(-1)^{\operatorname{codim}_{M} Y} c(\mathcal{L})^{\operatorname{dim} X}\left(\frac{c(T Y)}{c(\mathcal{L})^{\operatorname{dim} Y}} \cap \frac{[Y]}{c(\mathcal{L})^{\operatorname{codim}_{M} Y}}\right) \\
& =(-1)^{\operatorname{codim}_{M} Y} \frac{c(T Y)}{c(\mathcal{L})} \cap[Y]
\end{aligned}
$$

which yields the statement.

As an application, consider again the situation at the beginning of $\S 4.3: M_{1}$, $M_{2}$ are nonsingular hypersurfaces with contact scheme $Y$ (= singular scheme of $\left.M_{1} \cap M_{2}\right)$. Proposition IV.3 in $\S 4.3$ spells out a constraint imposed on the situation; in the particular case when $Y$ is nonsingular, Proposition IV.6 allows us to rewrite this in a particularly simple form:

Proposition IV.7. Assume $M_{1}, M_{2}$ are nonsingular hypersurfaces in a nonsingular ambient variety, and let $Y$ be their contact scheme. Assume $Y$ is nonsingular; then

$$
M_{1} \cdot Y=M_{2} \cdot Y .
$$

Proof. Let $X=M_{1} \cap M_{2}$. The last proposition can be used to compute $c_{\mathrm{SM}}(X)$ in two ways: considering $X$ as a hypersurface in $M_{1}$, with normal bundle $\mathcal{L}_{2}=$ 
$\left.\mathcal{O}\left(M_{2}\right)\right|_{X}$, or as a hypersurface in $M_{2}$, with normal bundle $\mathcal{L}_{1}=\left.\mathcal{O}\left(M_{1}\right)\right|_{X}$. This gives

$$
c_{F}(X)+(-1)^{\operatorname{codim}_{M} Y} \frac{c(T Y)}{c\left(\mathcal{L}_{2}\right)} \cap[Y]=c_{F}(X)+(-1)^{\operatorname{codim}_{M} Y} \frac{c(T Y)}{c\left(\mathcal{L}_{1}\right)} \cap[Y],
$$

and hence

$$
c\left(\mathcal{L}_{1}\right) \cap[Y]=c\left(\mathcal{L}_{2}\right) \cap[Y],
$$

from which the stated formula follows.

Again, the formula stated above should hold in $A_{*} Y$, although our argument only proves it in $A_{*}\left(M_{1} \cup M_{2}\right)$. This suffices however for some concrete applications. For example, say the ambient variety is a projective space, $d_{i}=\operatorname{deg} M_{i}$, and the contact scheme $Y$ of $M_{1}, M_{2}$ is nonsingular (as a scheme, not just as a set) and positive dimensional; then the statement is that necessarily $d_{1}=d_{2}$. It is easy to produce examples of hypersurfaces of the same degree and having nonsingular contact scheme: for instance, the quadrics

$$
\begin{aligned}
x^{2}+y^{2}+z^{2}+w^{2} & =0, \\
x^{2}+y^{2}+z^{2}+2 w^{2} & =0
\end{aligned}
$$

in $\mathbb{P}^{3}$ meet along a double conic, so have contact scheme equal to a nonsingular plane conic. Proposition IV.7 shows that no such example can be concocted with smooth hypersurfaces of different degrees.

\section{REFERENCES}

[Aluffi1] P. Aluffi, Singular schemes of hypersurfaces, Duke Math. J. 80 (1995), 325-351. MR 97b: 14057

[Aluffi2] P. Aluffi, MacPherson's and Fulton's Chern Classes of Hypersurfaces, I.M.R.N. (1994), 455-465. MR 96d:14004

[A-F] P. Aluffi, C. Faber, A remark on the Chern class of a tensor product, Manu. Math. 88 (1995), 85-86. MR 96e:14002

[B-S] J.-P. Brasselet, M.-H. Schwartz, Sur les classes de Chern d'un ensemble analytique complexe, Astérisque 82-83 (1981), 93-147. MR 83h:32011

[BDK] J.-L. Brylinski, A. Dubson, M. Kashiwara, Formule de l'indice pour les Modules Holonomes et obstruction d'Euler locale, C. R. Acad. Sci. Paris Sér. I Math. 293 (1981), 573-576. MR 83a:32010

[BFM] P. Baum, W. Fulton, R. MacPherson, Riemann-Roch for singular variety, Publ. Math. I.H.E.S. 45 (1975), 101-145. MR 54:317

[BMM] J. Briançon, P. Maisonobe, M. Merle, Localisation de systèmes différentiels, stratifications de Whitney et condition de Thom, Invent. Math. 117 (1994), 531-550. MR 95h:32043

[Fulton] W. Fulton, Intersection Theory, Springer Verlag, 1984. MR 85k:14004

[Ginsburg] V. Ginsburg, Characteristic varieties and vanishing cycles, Invent. Math. 84 (1986), 327-402. MR 87j:32030

[Kennedy] G. Kennedy, MacPherson's Chern classes of singular algebraic varieties, Comm. in Algebra 18 (1990), 2821-2839. MR 91h:14010

[Kwieciński] M. Kwieciński, Sur le transformé de Nash et la construction du graphe de MacPherson, Thèse, Université de Provence (1994).

[Lê-Mebkhout] Lê Dung Tráng and Z. Mebkhout, Variétés caractéristiques et variétés polaires, C. R. Acad. Sci. Paris Sér. I Math. 296 (1983), 129-132. MR 84g:32018

[MacPherson] R. MacPherson, Chern classes for singular algebraic varieties, Annals of Math. 100 (1974), 423-432. MR 50:13587

[Massey] D. Massey, Numerical invariants of perverse sheaves, Duke Math. J. 73 (1994), 307-369. MR 95e:32045 
[Nobile] A. Nobile, Some properties of the Nash blowing-up, Pacific J. of Math. 60 (1975), 297-305. MR 53:13217

[Parusiński1] A. Parusiński, A generalization of the Milnor number, Math. Ann. 281 (1988), 247-254. MR 89k:32023

[Parusiński2] A. Parusiński, Limits of tangent spaces to fibres and the $w_{f}$ condition, Duke Math. J. 72 (1993), 99-108. MR 94i:32059

[Sabbah] C. Sabbah, Quelque remarques sur la géométrie des espaces conormaux, Astérisque 130 (1985), 161-192. MR 87f:32031

[Schwartz] M.-H. Schwartz, Classes caractéristiques définies par une stratification d'une variété analytique complexe, C. R. Acad. Sci. Paris 260 (1965), 3262-3264, 35353537. MR 32:1727; MR 35:3707

[Silvotti] R. Silvotti, On a conjecture of Varchenko, Invent. Math. 126 (1996), 235-248. MR 98d:32038

[Suwa] T. Suwa, Classes de Chern des intersections complètes locales, C. R. Acad. Sci. Paris Sér. I Math. 324 (1997), 67-70. MR 97m:14003

Department of Mathematics, Florida State University, Tallahassee, Florida 32306

E-mail address: aluffi@math.fsu.edu 